\title{
Triggered massive star formation associated with the bubble HII region Sh2-39 (N5) ${ }^{\star}$
}

\author{
N. U. Duronea ${ }^{1}$, C. E. Cappa $^{1,2}$, L. Bronfman ${ }^{3}$, J. Borissova ${ }^{4,5}$, M. Gromadzki ${ }^{6,5,4}$, and M. A. Kuhn ${ }^{4,5}$ \\ ${ }^{1}$ Instituto Argentino de Radioastronomía, CONICET, CCT-La Plata, C.C.5., 1894, Villa Elisa, and CIC, Prov. de Bs. As., Argentina \\ e-mail: duronea@iar.unlp.edu.ar \\ 2 Facultad de Ciencias Astronómicas y Geofísicas, Universidad Nacional de La Plata, Paseo del Bosque s/n, 1900 La Plata, Argentina \\ 3 Departamento de Astronomía, Universidad de Chile, Casilla 36, Santiago de Chile, Chile \\ 4 Instituto de Física y Astronomía, Universidad de Valparaíso, Av. Gran Bretaña 1111, Playa Ancha, Valparaíso, Chile \\ 5 Millennium Institute of Astrophysics (MAS), Vicuña Mackenna 4860, 7820436 Macul, Santiago, Chile \\ 6 Warsaw University Astronomical Observatory, Al. Ujazdowskie 4, 00-478 Warszawa, Poland
}

Received 30 January 2017 / Accepted 1 June 2017

\begin{abstract}
Aims. We perform a multiwavelength analysis of the bubble HII region Sh2-39 (N5) and its environs with the aim of studying the physical properties of Galactic IR bubbles and exploring their impact in triggering massive star formation.

Methods. To analyze the molecular gas, we used $\mathrm{CO}(3-2)$ and $\mathrm{HCO}^{+}(4-3)$ line data obtained with the on-the-fly technique from the ASTE telescope. To study the distribution and physical characteristics of the dust, we made use of archival data from ATLASGAL, Herschel, and MSX, while the ionized gas was studied making use of an NVSS image. We used public WISE, Spitzer, and MSX point source catalogs to search for infrared candidate young stellar objects (YSOs) in the region. To investigate the stellar cluster [BDS2003]6 we used IR spectroscopic data obtained with the ARCoIRIS spectrograph, mounted on Blanco $4 \mathrm{~m}$ Telescope at CTIO, and new available IR $K$ s band observations from the VVVeXtended ESO Public Survey (VVVX).

Results. The new ASTE observations allowed the molecular gas component in the velocity range from $30 \mathrm{~km} \mathrm{~s}^{-1}$ to $46 \mathrm{~km} \mathrm{~s}^{-1}$, associated with Sh2-39, to be studied in detail. The morphology of the molecular gas suggests that the ionized gas is expanding against its parental cloud. We identified four molecular clumps, which were likely formed by the expansion of the ionization front, and determined some of their physical and dynamical properties. Clumps with $\mathrm{HCO}^{+}$and $870 \mu \mathrm{m}$ counterparts show evidence of gravitational collapse. We identified several candidate YSOs across the molecular component. Their spatial distribution and the fragmentation time derived for the collected layers of the molecular gas suggest that massive star formation might have been triggered by the expansion of the nebula via the collect and collapse mechanism. The spectroscopical distance obtained for the stellar cluster [BDS2003]6, placed over one of the collapsing clumps in the border of the HII region, reveals that this cluster is physically associated with the nebula and gives more support to the triggered massive star formation scenario. A radio continuum data analysis indicates that the nebula is older and expands at lower velocity than typical IR Galactic bubbles.
\end{abstract}

Key words. molecules data - infrared: ISM - stars: formation - HII regions - stars: massive

\section{Introduction}

Although massive star formation processes are still under debate (Zinnecker \& Yorke 2007; McKee \& Ostriker 2007), it is believed that they are born inside dense $\left(n \sim 10^{3-8} \mathrm{~cm}^{-3}\right)$, small $(R \sim 0.1-5 \mathrm{pc})$, and massive $\left(M \sim 100-1000 M_{\odot}\right)$ clumps of molecular gas and dust with high optical extinction. Understanding the formation and early stages of massive stars requires, therefore, a deep knowledge of the original physical conditions of the regions where they are born and their surroundings.

It is well-known that a considerable number of massive stars in our Galaxy can be formed by the action of HII regions over their parental molecular environment through processes like the collect-and-collapse mechanism $(C \& C$; Elmegreen \& Lada 1977) or the radiative driven implosion process (RDI; Lefloch \& Lazareff 1994). In recent years, the triggered star

\footnotetext{
* The ASTE data cubes and ARCoIRIS spectrum are only available at the CDS via anonymous ftp to

cdsarc.u-strasbg. fr (130.79.128.5) or via

http://cdsarc.u-strasbg.fr/viz-bin/qcat?J/A+A/606/A8
}

formation process, especially the $\mathrm{C} \& \mathrm{C}$ mechanism, has been studied extensively at the edges of many bubble-shaped HII regions such as Sh2-104, RCW 79, Sh2-212, RCW 120, Sh2-217, Sh2-90, Gum 31, and S 24 (Deharveng et al. 2003, 2008, 2009; Zavagno et al. 2006, 2010; Brand et al. 2011; Samal et al. 2014; Duronea et al. 2015; Cappa et al. 2016). Dense molecular clumps placed along the border of Galactic bubble HII regions are therefore among the most likely sites for stellar births, and hence, the regions to look for early stages of massive star formation. Keeping this in mind, it is instructive to study the interstellar medium adjacent to these objects since they can provide important information about the molecular environment where massive stars can be formed.

As part of a project aimed at studying the physical properties of IR bubbles and to better understand their influence in triggering massive star formation, we selected the northern nebula Sh2-39 (Sharpless 1959), which is a bubble nebula that was partially imaged in the Spitzer GLIMPSE survey, listed as N5 by Churchwell et al. (2006), and barely studied by Beaumont \& Williams (2010). The selection of this object was made taking into account the detection of the $\mathrm{CS}(2-1)$ line from 


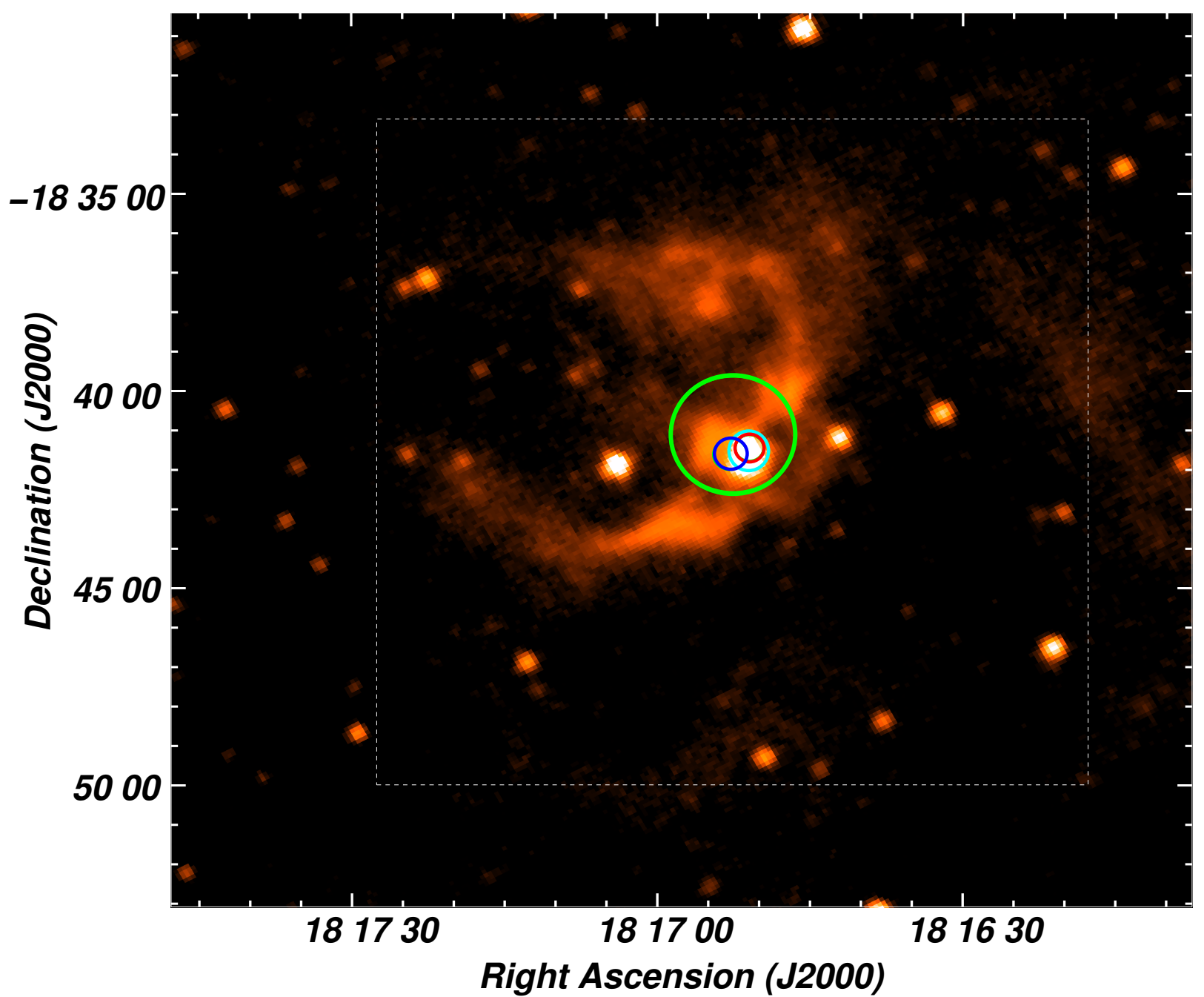

Fig. 1. MSX-A $(8.28 \mu \mathrm{m})$ emission of the IR bubble nebula N5 and its environs. The white dashed square indicates the area observed with the ASTE telescope. The position and beam size of the CS(2-1) line observation from Bronfman et al. (1996) is shown with the blue circle, while red and green circles show the position and beam size of the observations from Urquhart et al. (2011) and Lockman (1989), respectively (see text). The approximate location and size of the cluster [BDS2003]6 is also shown with the cyan circle.

the survey by Bronfman et al. (1996), ensuring the existence of high density molecular gas, which is a necessary condition for the massive star formation.

Here we present the very first multiwavelength study of Sh2-39 and its environs. We carried out observations of the $\mathrm{CO}(3-2)$ and $\mathrm{HCO}^{+}(4-3)$ lines with the ASTE telescope, aiming at studying in detail the spatial distribution and physical characteristics of the molecular gas associated with the nebula. To analyze the properties of the dust in the nebula and its surroundings we use images from the ATLASGAL and Herschel databases. To account for the interaction between the ionized and molecular gas and to determine the evolutionary status of the HII region, we use radio continuum data from the NVSS archives. This analysis also allows us to identify spots of dense molecular gas and dust where the massive star formation is developing, particularly the central region corresponding to the source IRAS 18139-1842, where star formation processes seem to be very active. In addition, we look for signatures of star formation in the surroundings using archival photometric data and we investigate a probable scenario of triggered star formation at the border of the nebula. We also study the characteristics of an identified stellar cluster projected onto the border of the bubble using new IR data. This provides some additional information that helps us to better understand the star formation activity in the nebula.

N5 is an open IR bubble of about $8^{\prime}$ in diameter centered at RA, Dec $(\mathrm{J} 2000)=18^{\mathrm{h}} 17^{\mathrm{m}} 02^{\mathrm{s}},-18^{\circ} 40^{\prime} 19^{\prime \prime}$, coincident with the HII region Sh2-39, and located at a distance of $4.1 \mathrm{kpc}$ (Bronfman et al. 1996; Faúndez et al. 2004). In Fig. 1 we present a $8.28 \mu \mathrm{m}$ (MSX-A) emission image of the nebula, which has a typical arc-shaped morphology in the mid-IR wavelengths. At wavelengths of about $\sim 8 \mu \mathrm{m}$ most of the emission originates in strong features of PAH molecules, which are considered to be good tracers of warm UV-irradiated photodissociation regions (PDR; Hollenbach \& Tielens 1997). Since these complex molecules are destroyed inside the ionized gas of an HII region, they indicate the limits of the ionization front and delineate the boundaries of the bubble nebula, giving a glimmer of the location of the parental molecular gas against which N5 is likely expanding. The open morphology of the IR nebula suggests that Sh2-39 is the classical HII region density bounded toward the outer side (eastward) and ionization bounded toward the molecular cloud (westward).

The nebula is believed to be linked to the IR sources IRAS 18139-1842 and IRAS 18139-1839, which both have 
characteristics of massive young stellar objects (see Sect. 6). The CS(2-1) line was detected by Bronfman et al. (1996) at a velocity ${ }^{1}$ of $40.2 \mathrm{~km} \mathrm{~s}^{-1}$ in the direction of IRAS $18139-1842$. $\mathrm{NH}_{3}$ line and $\mathrm{H}_{2} \mathrm{O}$ maser emission, which are believed to be excellent indicators of early stages of massive star formation, were also detected toward this IRAS source (Sunada et al. 2007; Urquhart et al. 2011), while radio recombination line observations $(\mathrm{H} 87 \alpha$ and $\mathrm{H} 88 \alpha)$ show emission at the same position at a velocity of $41 \mathrm{~km} \mathrm{~s}^{-1}$ (Lockman, 1989, see Fig. 1), which indicates the existence of high density ionized gas at the same radial velocity as the molecular gas. The radio source G012.431701.1112, presumably associated with IRAS 18139-1842, was studied by Urquhart et al. (2009) who classified the source as a classical HII region.

Regarding the stellar content in the region of the nebula, using the 2MASS catalog Bica et al. (2003) identified the IR cluster candidate [BDS2003]6, of about 0'8 in size, coincident with the position of IRAS 18139-1842 (see Fig. 1). The presence of this stellar cluster projected onto the IR emission is further strong evidence for massive star formation in the border of the bubble.

\section{Observations and complementary data}

\subsection{Molecular data}

The molecular observations were carried out in August 2015 with the $10 \mathrm{~m}$ Atacama Submillimeter Telescope Experiment (ASTE; Ezawa et al. 2004, 2008). We used DASH345, a twosideband single-polarization heterodyne receiver, tunable in LO frequency range from $327 \mathrm{GHz}$ to $370 \mathrm{GHz}$ at observable frequency range from $321 \mathrm{GHz}$ to $376 \mathrm{GHz}$. The XF digital spectrometer was set to a bandwidth and spectral resolution of $128 \mathrm{MHz}$ and $125 \mathrm{KHz}$, respectively. The spectral velocity resolution was $0.11 \mathrm{~km} \mathrm{~s}^{-1}$, the half power beamwidth (HPBW) is $\sim 22^{\prime \prime}$ and the main beam efficiency $\left(\eta_{\mathrm{mb}}\right)$ is 0.65 . Observations were made using the on-the-fly (OTF) mode with two orthogonal scan directions along RA and Dec(J2000) centered on $\mathrm{RA}, \operatorname{Dec}(\mathrm{J} 2000)=\left(18^{\mathrm{h}} 16^{\mathrm{m}} 51.4^{\mathrm{s}},-18^{\circ} 41^{\prime} 40^{\prime \prime}\right)$. We observed simultaneously the lines $\mathrm{CO}(3-2)(345.796 \mathrm{GHz})$ and $\mathrm{HCO}^{+}(4-3)$ (356.734) in a region of $\sim 17^{\prime} \times 17^{\prime}$ (see Fig. 1). The spectra were reduced with NOSTAR ${ }^{2}$ using the standard procedure.

\subsection{Infrared spectroscopic data}

The brightest star projected at the center of [BDS2003] 6 (2MASSJ 18165113-1841488) was observed on August 2016 with the Astronomy Research using the Cornell Infra Red Imaging Spectrograph (ARCoIRIS), a cross-dispersed, single-object, longslit, infrared imaging spectrograph, mounted on Blanco $4 \mathrm{~m}$ Telescope, CTIO. The spectra cover a simultaneous wavelength range of 0.80 to $2.47 \mu \mathrm{m}$, at a spectral resolution of about 3500 $\lambda / \Delta \lambda$, encompassing the entire $z Y J H K$ photometric range. The spectrum was taken with four exposures, each $120 \mathrm{~s}$ in an ABBA dithering pattern for proper subtraction of sky background. The average airmass was 1.03 . The HD 163336 tellluric A0 V standard is observed immediately after target. We reduced these data using the Spextool IDL package (version 4.1; Cushing et al. 2004), which is new suite of data reduction algorithms, that are

\footnotetext{
1 All the velocities in this work are referred to the local standard of rest (LSR).

2 http://alma.mtk.nao.ac.jp/aste/guide/otf/reduct-e. html
}

specifically designed for the data format and characteristics of ARCoIRIS by Dr. Katelyn Allers ${ }^{3}$. Telluric correction and flux calibration of the post-extraction spectra are achieved through the xtellcorr IDL package (Vacca et al. 2003).

\subsection{Archival data}

The data described above were complemented with several archival data sets:

- Infrared data: a) Images of ATLASGAL at $870 \mu \mathrm{m}$ (345 GHz) (Schuller et al. 2009). This survey covers the inner Galactic plane, $l=300^{\circ}$ to $60^{\circ},|b| \leq 1 .^{\circ} 5$, with a rms noise in the range $0.05-0.07 \mathrm{Jy}^{\text {beam }^{-1}}$. The Large APEX BOlometer CAmera (LABOCA) used for these observations is a 295-pixel bolometer array developed by the Max-PlanckInstitut fur Radioastronomie (Siringo et al. 2007). The beam size at $870 \mu \mathrm{m}$ is $19^{\prime \prime}$.2. b) The new project, The VVV eXtended ESO Public Survey (VVVX; Hempel et al., in prep.), was approved as an extension of the VISTA Variables in the Via Lactea (VVV; Minniti et al. 2010; Saito et al. 2012) to enhance its long-lasting legacy. The VVVX Survey will extend the VVV time baseline and will cover additional to VVV 1700 sq/degrees in the southern sky $(7 \mathrm{~h}<\mathrm{RA}<19 \mathrm{~h})$. The VVVX started in 2016 and the region of N5 was observed in $K$ s band in August 2016. We retrieved 10 images from Cambridge Astronomical Survey Unit (CASU) VIRCAM pipeline (Irwin et al. 2004), each of which has a $4 \mathrm{~s}$ exposure time covering area of $11^{\prime} \times 11^{\prime}$. The images were combined using standard IRAF procedures. c) Images from the Herschel ${ }^{4}$ Infrared GALactic (Hi-GAL) plane survey key program (Molinari et al. 2010). We used images from the SPIRE archive data (Griffin et al. 2010) at $250 \mu \mathrm{m}$ and $350 \mu \mathrm{m}$ with beam sizes of $18^{\prime \prime}$ and $25^{\prime \prime}$, respectively. We retrieved images processed at level 2.5 from the NASA/IPAC database $^{5}$. d) Infrared data at $8.28 \mu \mathrm{m}$ retrieved from the Midcourse Space Experiment (MSX) ${ }^{6}$ (Price et al. 2001). The image has a spatial resolution of $20^{\prime \prime}$.

- Radio continuum image at $1.4 \mathrm{GHz}$ obtained from the NRAO Very Large Aray (VLA) Sky Survey ${ }^{7}$ (NVSS; Condon et al. 1998). The images have a spatial resolution of $45^{\prime \prime}$ and a rms noise of $0.45 \mathrm{mJy}^{-1}$ beam $^{-1}$ (Stokes $I$ ).

- To investigate the existence of candidate YSOs projected onto the region we analyzed infrared point sources from the MSX survey (Price et al. 2001), the Galactic Plane Survey (GPS) of the UKIRT Infrared Deep Sky Survey (UKIDSS, Lucas et al. 2008), the Spitzer survey (Fazio et al. 2004), and the Wide-field Infrared Survey Explorer (WISE; Wright et al. 2010).

\section{Molecular gas analysis}

\subsection{Spatial distribution of the molecular gas and identification of clumps}

In Fig. 2 we show the $\mathrm{CO}(3-2)$ spectrum averaged over a region $\sim 12^{\prime} \times 12^{\prime}$ in size around the center of the nebula

\footnotetext{
3 http://www.ctio.noao.edu/noao/node/9701

4 Herschel is an ESA space observatory with science instruments provided by European-led Principal Investigator consortia and with important participation from NASA (http://www. cosmos.esa.int/web/ herschel/science-archive).

5 http://irsa.ipac.caltech.edu/frontpage/

6 http://irsa.ipac.caltech.edu/Missions/msx.html

7 http://www.cv.nrao.edu/nvss/
} 


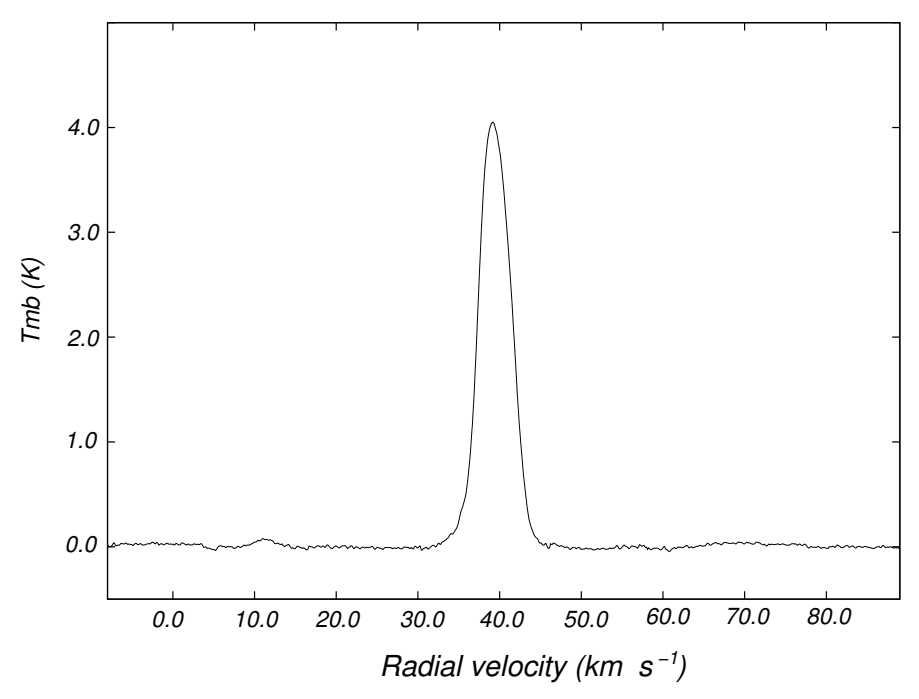

Fig. 2. Averaged spectrum obtained within a region of $\sim 12^{\prime} \times 12^{\prime}$ centered on RA, Dec $(\mathrm{J} 2000)=18^{\mathrm{h}} 17^{\mathrm{m}} 02.1^{\mathrm{s}},-18^{\circ} 40^{\prime} 19^{\prime \prime}$.

$\left(\mathrm{RA}, \operatorname{Dec}(\mathrm{J} 2000)=18^{\mathrm{h}} 17^{\mathrm{m}} 02.1^{\mathrm{s}},-18^{\circ} 40^{\prime} 19^{\prime \prime}\right)$. The molecular emission appears concentrated in only one component between $\sim 30 \mathrm{~km} \mathrm{~s}^{-1}$ and $46 \mathrm{~km} \mathrm{~s}^{-1}$ with a peak emission around $39.5 \mathrm{~km} \mathrm{~s}^{-1}$. This velocity is almost coincident with the peak velocity of the CS(2-1) line reported by Bronfman et al. (1996) and the HH87 $\alpha$ recombination line reported by Lockman (1989) toward IRAS 18139-1842.

In the panel a of Fig. 3 we show the integrated $\mathrm{CO}(3-2)$ emission distribution in the mentioned velocity range. As expected, the molecular gas appears concentrated toward the western and southern borders of the IR nebula, while no significant molecular emission is detected toward the eastern and northeastern borders. This is in line with the proposed scenario of an HII region bounded by density to the east and ionization to the west. The morphology and location of the molecular gas and radio continuum emission (see Sect. 5) suggest that the ionized gas is expanding against the molecular cloud, probably in a blister-type HII region scenario (Israel 1978). The morphology, distribution, and location of the molecular gas with respect to the near IR emission is similar to many other in Galactic IR bubbles found in the literature.

Although morphological evidence suggests that the molecular cloud is expanding, we performed several tests to detect expanding motions in the molecular gas (not shown here) with no conclusive results. This very likely indicates that the bubble is evolved or else is expanding at a low rate. This scenario is further tested in Sect. 7.2 by analyzing the properties of the ionized gas.

The molecular emission is not uniformly distributed and several condensations can be distinguished in the whole structure. These condensations, referred hereafter to as "clumps" (Blitz 1993), must be identified and their physical properties must be estimated if a study of the molecular gas in Sh2-39 is to be carried out. To identify the CO clumps and unambiguously ascertain their geometrical and physical properties, we made use of the clumpfind algorithm (Williams et al. 1994). We were left only with the brightest clumps which are, in turn, those adjacent to the bubble. These clumps are the places where star formation seems to be taking place (see Sect. 6) and were very likely formed in the collected layers of the molecular gas as a result of the expansion of the nebula against the parental molecular cloud. The location of the clumps, labeled from 1 to 4 , is depicted in Fig. 3a and their observational parameters are indicated in Table 1. The area of the clumps in the $\mathrm{CO}$ emission $\left(A_{\text {clump }}^{\mathrm{CO}}\right.$; Col. 4 in Table 1$)$ was estimated with the clumpfind algorithm. The peak temperatures $\left(T_{\text {peak }}^{\mathrm{CO}}\right.$; Col. 5 in Table 1) and the velocity of the $\mathrm{CO}$ emission peak $\left(v_{\text {peak }}^{\mathrm{CO}}\right.$; Col. 6 in Table 1$)$ were estimated by Gaussian fitting in spectra obtained in the direction of the maximum emission of each clump. The line width of the $\mathrm{CO}$ line for each clump ( $\Delta V^{\mathrm{CO}}$; Col. 7 in Table 1 ) was obtained as $2 \times \sqrt{2 \ln 2} \times \sigma_{\text {gauss }}$.

In order to provide a better visual display of the dynamics of the molecular gas component, in panels b to i of Fig. 3 we show the channel maps of $\mathrm{CO}(3-2)$ in velocity intervals of $2 \mathrm{~km} \mathrm{~s}^{-1}$, overlaid onto the $8.28 \mu \mathrm{m}$ (in red) and $870 \mu \mathrm{m}$ (in green) emissions. In the velocity interval from $30 \mathrm{~km} \mathrm{~s}^{-1}$ to $34 \mathrm{~km} \mathrm{~s}^{-1}$ (panels b and c) clump 2 becomes noticeable, achieving its maximum emission temperature in the velocity interval from $36 \mathrm{~km} \mathrm{~s}^{-1}$ to $38 \mathrm{~km} \mathrm{~s}^{-1}$ (panel e). This clump is detected up to a velocity of $39 \mathrm{~km} \mathrm{~s}^{-1}$ (not shown here), where it is mixed with the emission of clumps 3 and 1 . Clumps 1 and 4 become detected at velocities between $32 \mathrm{~km} \mathrm{~s}^{-1}$ to $36 \mathrm{~km} \mathrm{~s}^{-1}$ (panels $\mathrm{c}$ and d). In this velocity interval a faint extended molecular emission appears bordering the western and northwestern side of the nebula. This structure is detected up to a velocity of $\sim 42 \mathrm{~km} \mathrm{~s}^{-1}$. Clumps 1 and 4 achieve their maximum emission temperature in the velocity interval from $40 \mathrm{~km} \mathrm{~s}^{-1}$ from $42 \mathrm{~km} \mathrm{~s}^{-1}$ (panel g) and from $38 \mathrm{~km} \mathrm{~s}^{-1}$ to $40 \mathrm{~km} \mathrm{~s}^{-1}$ (panel f), respectively. Both clumps are detected until a velocity of $\sim 45 \mathrm{~km} \mathrm{~s}^{-1}$. Clump 3 is noticed in the velocity interval from $36 \mathrm{~km} \mathrm{~s}^{-1}$ to $44 \mathrm{~km} \mathrm{~s}^{-1}$ (panels e to $\mathrm{h}$ ), achieving its maximum emission temperature in the velocity interval from $38 \mathrm{~km} \mathrm{~s}^{-1}$ to $40 \mathrm{~km} \mathrm{~s}^{-1}$ (panel f). In the velocity interval from $38 \mathrm{~km} \mathrm{~s}^{-1}$ to $46 \mathrm{~km} \mathrm{~s}^{-1}$ (panels $\mathrm{f}$ to i) the southeastern border of the nebula is surrounded by a filamentary molecular structure detected up to a velocity of $\sim 47 \mathrm{~km} \mathrm{~s}^{-1}$. Unlike IRAS 18139-1842, which seems to be physically related with clump 1, IRAS 18139-1839 does not seem to have an associated molecular gas component. No substantial molecular emission is detected either toward the center or the east of the nebula, which suggests a density gradient in the region.

In the lower right corner of Fig. $3 \mathrm{i}$ we show an enlargement of the central region showing the emission of the $\mathrm{HCO}^{+}(4-3)$ line (black contours) in the velocity range from $35.5 \mathrm{~km} \mathrm{~s}^{-1}$ to $42.4 \mathrm{~km} \mathrm{~s}^{-1}$. Two molecular structures (or clumps) are detected with centers at RA, Dec $(\mathrm{J} 2000)=18^{\mathrm{h}} 16^{\mathrm{m}} 51.4^{\mathrm{s}},-18^{\circ} 41^{\prime} 36^{\prime \prime}$ and RA, Dec $(J 2000)=18^{\mathrm{h}} 16^{\mathrm{m}} 51.6^{\mathrm{s}},-18^{\circ} 43^{\prime} 09^{\prime \prime}$. Their location and size suggest that these clumps are the $\mathrm{HCO}^{+}$counterparts of $\mathrm{CO}$ clumps 1 and 2 . The detection of the $\mathrm{HCO}^{+}(4-3)$ line and $870 \mu \mathrm{m}$ emission indicate that these clumps have high densities making them potential good candidates sites to form massive stars. The location of the stellar cluster [BDS2003]6 in the direction of clump 1 (see Fig. 1) gives more support to this scenario.

For the case of the $\mathrm{HCO}^{+}$clumps, their areas $\left(A_{\text {clump }}^{\mathrm{HCO}+}\right.$; Col. 8 in Table 1) were estimated using the contour level of $0.07 \mathrm{~K} \mathrm{~km} \mathrm{~s}^{-1}(\sim 8 \mathrm{rms})$. The peak temperature of the $\mathrm{HCO}^{+}$ clumps $\left(T_{\text {peak }}^{\mathrm{HCO}^{+}}\right.$; Col. 9 , in 1$)$ and the velocity of the $\mathrm{HCO}^{+}$emission peak $\left(v_{\text {peak }}^{\mathrm{HCO}+}\right.$; Col. 10 in Table 1$)$ were estimated by Gaussian fitting in spectra obtained toward the maximum emission of each clump. The line width of the $\mathrm{HCO}^{+}$line $\left(\Delta V^{\mathrm{HCO}^{+}}\right)$is indicated in Col. 11 in Table 1.

\subsection{Physical properties of the clumps}

The deconvolved effective radius of the clumps derived from the $\mathrm{CO}$ and $\mathrm{HCO}^{+}$lines, $R_{\mathrm{D}}^{\mathrm{CO}}$ and $R_{\mathrm{D}}^{\mathrm{HCO}+}($ Cols. 2 and 6 in Table 2) 
N. U. Duronea et al.: Triggered massive star formation associated with the bubble HII region Sh2-39 (N5)

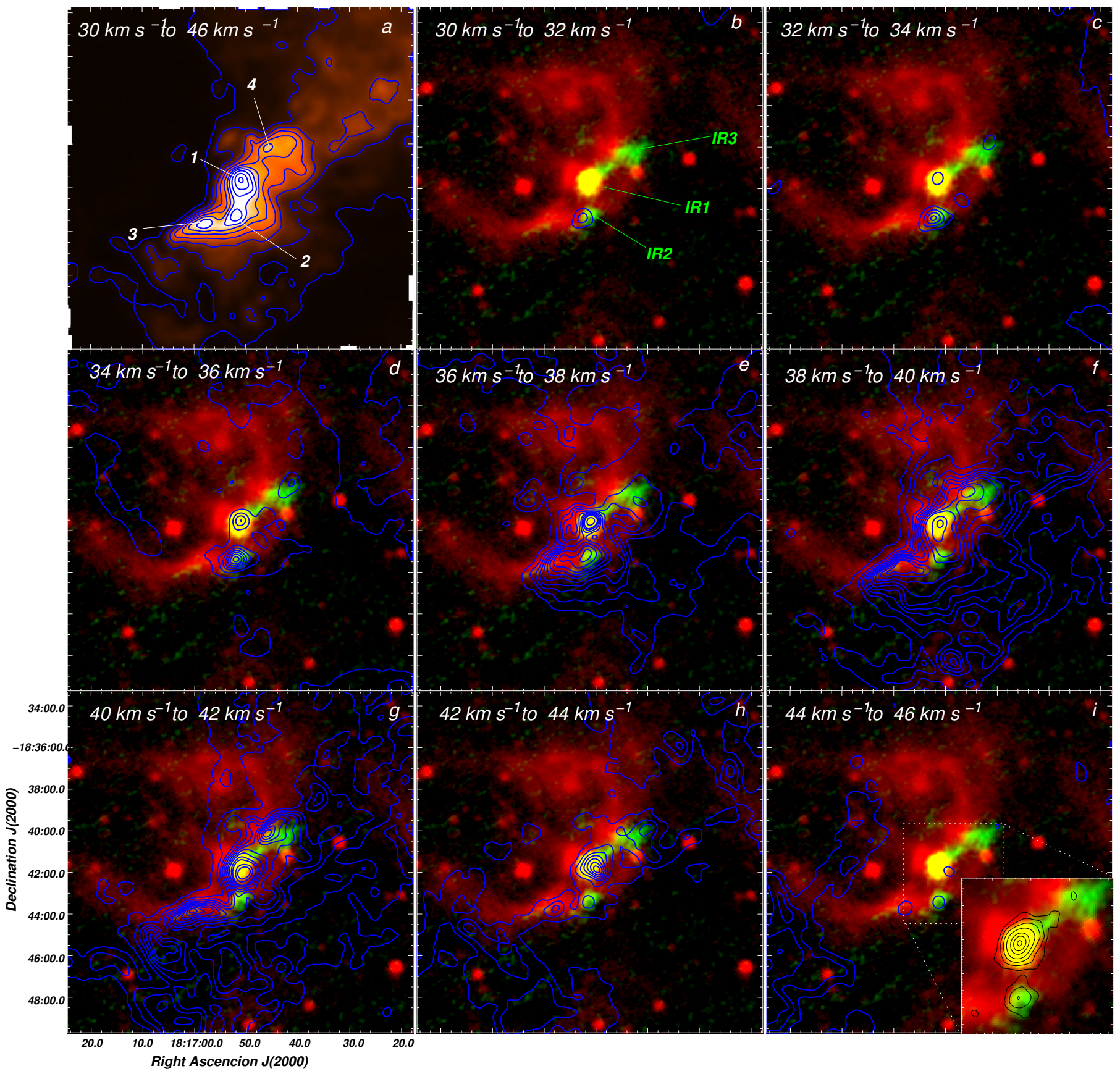

Fig. 3. Panel a: $\mathrm{CO}(3-2)$ emission in the velocity range from 30 to $46 \mathrm{~km} \mathrm{~s}^{-1}$. Contour levels go from $0.3 \mathrm{~K} \mathrm{~km} \mathrm{~s}^{-1}$ ( $\left.\sim 20 \mathrm{rms}\right)$ to $3.3 \mathrm{~K} \mathrm{~km} \mathrm{~s}{ }^{-1}$ in steps of $0.5 \mathrm{~K} \mathrm{~km} \mathrm{~s}^{-1}$ and from $4 \mathrm{~K} \mathrm{~km} \mathrm{~s}^{-1}$ in steps of $1 \mathrm{~K} \mathrm{~km} \mathrm{~s}^{-1}$. White numbers indicate the identified molecular clumps (see text). Panels $b$ : to i) channel maps of $\mathrm{CO}(3-2)$ in intervals of $2 \mathrm{~km} \mathrm{~s}^{-1}$ (blue contours) superimposed on the $8.28 \mu \mathrm{m} \mathrm{MSX-A} \mathrm{emission} \mathrm{(red} \mathrm{color} \mathrm{scale)} \mathrm{and} 870 \mu \mathrm{m}$ ATLASGAL emission (green color scale). Contours go from $0.2 \mathrm{~K} \mathrm{~km} \mathrm{~s}^{-1}\left(\sim 8 \mathrm{rms}\right.$ ) to $9.2 \mathrm{~K} \mathrm{~km} \mathrm{~s}^{-1}$ in steps of $1 \mathrm{~K} \mathrm{~km} \mathrm{~s}^{-1}$, and from $11 \mathrm{~K} \mathrm{~km} \mathrm{~s}^{-1}$ in steps of $2 \mathrm{~K} \mathrm{~km} \mathrm{~s}^{-1}$. Infrared sources identified in the $870 \mu \mathrm{m}$ emission (see Sect. 4) are indicated in panel $b$ in green. A zoom of the central region with the emission of the $\mathrm{HCO}^{+}(4-3)$ line (black contours) in the velocity interval from $36.5 \mathrm{~km} \mathrm{~s}^{-1}$ to $42.4 \mathrm{~km} \mathrm{~s}{ }^{-1}$ is shown in the lower right corner of panel $i$. $\mathrm{HCO}^{+}$contour levels are $0.07(\sim 8 \mathrm{rms}), 0.12,0.21,0.35,0.6,0.8$, and $1.0 \mathrm{~K} \mathrm{~km} \mathrm{~s}^{-1}$.

Table 1. Observed parameters of the $\mathrm{CO}$ and $\mathrm{HCO}^{+}$clumps.

\begin{tabular}{ccccccccccc}
\hline \hline clump & $\begin{array}{c}\mathrm{RA} \\
(\mathrm{h} \mathrm{m} \mathrm{s})\end{array}$ & $\begin{array}{c}\text { Dec } \\
\left({ }^{\prime}{ }^{\prime \prime}\right)\end{array}$ & $\begin{array}{c}A_{\text {clump }}^{\mathrm{CO}} \\
\left(10^{-7} \text { ster }\right)\end{array}$ & $\begin{array}{c}T_{\text {peak }}^{\mathrm{CO}}(\mathrm{K}) \\
(16\end{array}$ & $\begin{array}{c}v_{\text {peak }}^{\mathrm{CO}} \\
\left(\mathrm{km} \mathrm{s}^{-1}\right)\end{array}$ & $\begin{array}{c}\Delta V^{\mathrm{CO}} \\
\left(\mathrm{km} \mathrm{s}^{-1}\right)\end{array}$ & $\begin{array}{c}A_{\text {clump }}^{\mathrm{HCO}+} \\
\left(10^{-7} \text { ster }\right)\end{array}$ & $\begin{array}{c}T_{\text {peak }}^{\mathrm{HCO}} \\
(\mathrm{K})\end{array}$ & $\begin{array}{c}v_{\text {peak }}^{\mathrm{HCO}+} \\
\left(\mathrm{km} \mathrm{s}^{-1}\right)\end{array}$ & $\begin{array}{c}\Delta V^{\mathrm{HCO}+} \\
\left(\mathrm{km} \mathrm{s}^{-1}\right)\end{array}$ \\
\hline 1 & 181650.8 & -184140 & 3.9 & 10.6 & 38.3 & 6.5 & 1.9 & 1.1 & 40.1 \\
2 & 181651.8 & -184318 & 6.7 & 6.3 & 38.7 & 6.6 & 0.8 & 0.3 & 38.5 \\
3 & 181657.9 & -184339 & 4.5 & 7.2 & 35.1 & 5.9 & - & - & - \\
4 & 181645.8 & -184011 & 6.1 & 8.5 & 35.5 & 3.8 & - & - & - \\
\hline
\end{tabular}


Table 2. Physical properties derived for the $\mathrm{CO}$ and $\mathrm{HCO}^{+}$clumps.

\begin{tabular}{ccccccccc}
\hline \hline clump & $\begin{array}{c}R_{\mathrm{D}}^{\mathrm{CO}} \\
(\mathrm{pc})\end{array}$ & $\begin{array}{c}N\left(\mathrm{H}_{2}\right) \\
\left(10^{22} \mathrm{~cm}^{-2}\right)\end{array}$ & $\begin{array}{c}M_{\mathrm{int}}\left(\mathrm{H}_{2}\right) \\
\left(10^{3} M_{\odot}\right)\end{array}$ & $\begin{array}{c}n_{\left(\mathrm{H}_{2}\right)} \\
\left(10^{3} \mathrm{~cm}^{-3}\right)\end{array}$ & $\begin{array}{c}R_{\mathrm{D}}^{\mathrm{HCO}^{+}} \\
(\mathrm{pc})\end{array}$ & $\begin{array}{c}N\left(\mathrm{HCO}^{+}\right) \\
\left(10^{12} \mathrm{~cm}^{-2}\right)\end{array}$ & $\begin{array}{c}M_{\mathrm{LTE}} \\
\left(10^{3} M_{\odot}\right)\end{array}$ & $\begin{array}{c}M_{\mathrm{vir}} \\
\left(10^{3} M_{\odot}\right)\end{array}$ \\
\hline 1 & 1.4 & 1.3 & 2.0 & 3.3 & 1.0 & $1.0-2.3$ & $1.3-3.2$ & $1.3-2.1$ \\
2 & 1.9 & 0.9 & 2.2 & 1.5 & 0.6 & $0.4-1.0$ & $0.5-1.5$ & $0.6-0.9$ \\
3 & 1.5 & 0.8 & 1.4 & 1.8 & - & - & - & - \\
4 & 1.8 & 0.9 & 1.9 & 1.6 & - & - & - & - \\
\hline
\end{tabular}

were calculated as

$R_{\mathrm{D}}=\sqrt{R_{\mathrm{eff}}^{2}-\mathrm{HPBW}^{2} / 4}$,

where $R_{\text {eff }}$ is the effective radii of the clumps $\left(R_{\text {eff }}=\sqrt{A_{\text {clump }} / \pi}\right)$ calculated in both lines and HPBW is the half-power beam width of the instrument.

To estimate the mass of the clumps we use the relation between the $\mathrm{H}_{2}$ column density $\left(N\left(\mathrm{H}_{2}\right)\right.$; Col. 3 in Table 2$)$ and the $\mathrm{CO}$ integrated emission

$N\left(\mathrm{H}_{2}\right)=X \times \int T_{\mathrm{mb}}(\mathrm{CO}) \mathrm{d} v$,

where $X$ is an empirical factor that has been shown to be roughly constant for Galactic molecular clouds. For the ${ }^{12} \mathrm{CO}(1-0)$ line the $X$ value is about $1.9 \times 10^{20} \mathrm{~cm}^{-2}\left(\mathrm{~K} \mathrm{~km} \mathrm{~s}^{-1}\right)^{-1}$ (Strong \& Mattox 1996). Since we use the integrated intensity emission of the $\mathrm{CO}(3-2)$ line, we need to adjust the value of $N\left(\mathrm{H}_{2}\right)$ using a correcting factor of $\sim 0.7$ (Oka et al. 2012). Then, the total integrated hydrogen mass $\left(M_{\text {int }}\left(\mathrm{H}_{2}\right)\right.$, Col. 4 in Table 2) is calculated using

$M_{\text {int }}\left(\mathrm{H}_{2}\right)=\mu m_{\mathrm{H}} \sum \mathrm{A}_{\text {clump }} \frac{N\left(\mathrm{H}_{2}\right)}{0.7} d^{2}$,

where $\mu$ is the mean molecular weight, which is assumed to be equal to 2.8 after allowance of a relative helium abundance of $25 \%$ by mass, $m_{\mathrm{H}}$ is the hydrogen atom mass, $A_{\text {clump }}$ is the area subtended by the CO clump (see Table 1), and $d$ is the kinematical distance (assumed to be $4.1 \mathrm{kpc})$. The volume density $\left(n_{\left(\mathrm{H}_{2}\right)}\right.$, Col. 5 in Table 2) was derived assuming spherical geometry for each clump.

For the case of clumps 1 and 2, we also obtained the mass and volume density using the $\mathrm{HCO}^{+}$emission line. Assuming local thermodinamic equilibrium (LTE) the $\mathrm{HCO}^{+}$column density can be derived from

$N\left(\mathrm{HCO}^{+}\right)=5.85 \times 10^{10} \mathrm{e}^{\left(25.7 / T_{\text {exc }}\right)} \frac{T_{\text {exc }}+0.71}{1-\mathrm{e}^{-\left(17.12 / T_{\text {exc }}\right)}} \int \tau \mathrm{d} v$,

where $\tau$ is the optical depth of the $\mathrm{HCO}^{+}$line. As excitation temperatures, $T_{\text {exc }}$, we used a conservative range $20-50 \mathrm{~K}$, which is typically used for both low-mass and massive star forming regions. Assuming that the $\mathrm{HCO}^{+}(4-3)$ line is optically thin, we can use the approximation

$\int \tau \mathrm{d} v=\frac{1}{J\left(T_{\text {exc }}\right)-J\left(T_{\mathrm{bg}}\right)} \times \int T_{\mathrm{mb}}\left(\mathrm{HCO}^{+}\right) \mathrm{d} v$,

where

$J(T)=\frac{h v / k}{\mathrm{e}^{(h v / k T)}-1}$

and $T_{\mathrm{bg}}$ is the background temperature $(\sim 2.7 \mathrm{~K})$. The LTE mass $\left(M_{\text {LTE }}\right.$, Col. 8 in Table 2$)$ can be then obtained assuming a mean fractional abundance $X\left(\mathrm{HCO}^{+}\right)=5 \times 10^{-11}$ (Cortes et al. 2010; Cortes 2011) to obtain $N\left(\mathrm{H}_{2}\right)$ and using Eq. (3) without the correcting factor 0.7 .

Considering only gravitational and internal pressure, neglecting support of magnetic fields or internal heating sources, the virialized molecular mass $\left(M_{\mathrm{vir}}\right.$; Col. 9 in Table 2$)$ can be estimated using the $\mathrm{HCO}^{+}$line (assumed to be optically thin) from

$$
M_{\mathrm{vir}}=k R^{\mathrm{HCO}^{+}}\left(\Delta V^{\mathrm{HCO}+}\right)^{2}
$$

(MacLaren et al. 1988), where the constant $\mathrm{k}$ depends on the geometry of the density structure, that is 126 or 190 according to the density-radius distribution $n \propto r^{-2}$ or $n \propto r^{-1}$, respectively. Virial masses were only derived for clumps 1 and 2 since no $\mathrm{HCO}^{+}$emission was detected for clumps 3 and 4 .

\section{Submillimeter emission}

When an HII region expands, molecular gas and dust accumulate behind the ionization front, forming shells of dense molecular gas surrounding the ionization front. Eventually, these shells become massive and could contain cold dust that radiates in the (sub-)millimeter range. The continuum emission at $870 \mu \mathrm{m}$ is probably one of the most reliable tracers of the dense molecular gas. It is usually dominated by the thermal emission from dust contained in dense material (e.g., dense star forming clumps and filaments) and provides a powerful tool for probing some basic physical properties of dense clumps (column density of molecular hydrogen, mass, etc.) that are needed to unveil the physical conditions in regions where stars can form.

In Fig. 3 (panels b to i) we show the $870 \mu \mathrm{m}$ emission in green color scale. Three bright structures can be discerned centered approximately at RA, Dec $(J 2000)=18^{\mathrm{h}} 16^{\mathrm{m}} 51.7^{\mathrm{s}}$, $-18^{\circ} 41^{\prime} 35^{\prime \prime}$, RA, Dec $(\mathrm{J} 2000)=18^{\mathrm{h}} 16^{\mathrm{m}} 51.0^{\mathrm{s}},-18^{\circ} 43^{\prime} 08^{\prime \prime}$, and $\mathrm{RA}, \operatorname{Dec}(\mathrm{J} 2000)=18^{\mathrm{h}} 16^{\mathrm{m}} 44.8^{\mathrm{s}},-18^{\circ} 40^{\prime} 19^{\prime \prime}$. Their position, size, and shape suggest that these sources are the IR counterparts of CO clumps 1, 2, and 4. For the sake of the analysis, we refer to these submillimeter sources as IR1, IR2, and IR3 (see panel b of Fig. 3).

In Table 3 we present some physical and geometrical properties derived for the sources. The total $\left(\mathrm{H}_{2}+\right.$ dust $)$ mass of the clumps ( $M_{\text {tot }}$, Col. 9 in Table 3 ) was calculated from their integrated $870 \mu \mathrm{m}$ flux density emission inside the $3 \mathrm{rms}$ level $\left(S_{870}\right.$, Col. 4 in Table 3), assuming that the emission is optically thin, and using the equation

$M_{\mathrm{tot}}=R \frac{S_{870} d^{2}}{\kappa_{870} B_{870}\left(\bar{T}_{\text {dust }}\right)}$

(Hildebrand 1983), where $R$ is the gas-to-dust ratio (assumed to be $~ 186$; Jenkins 2004; Draine et al. 2007), $d$ is the distance, $\kappa_{870}$ is the dust opacity per unit mass at $870 \mu \mathrm{m}$ assumed to be $1.0 \mathrm{~cm}^{2} \mathrm{~g}^{-1}$ (estimated for dust grains with thin ice mantles in cold clumps; Ossenkopf \& Henning 1994), and $B_{870}\left(\bar{T}_{\text {dust }}\right)$ is the 
Table 3. Properties derived for the submillimeter sources.

\begin{tabular}{|c|c|c|c|c|c|c|c|c|c|c|c|}
\hline Source & $\begin{array}{c}\mathrm{RA} \\
(\mathrm{h} \mathrm{m} \mathrm{s})\end{array}$ & $\begin{array}{c}\text { Dec } \\
\left({ }^{\prime \prime} \prime^{\prime \prime}\right)\end{array}$ & $\begin{array}{l}S_{870} \\
(\mathrm{Jy})\end{array}$ & $\begin{array}{c}I_{870} \\
(\mathrm{Jy} / \text { beam })\end{array}$ & $\begin{array}{l}R_{\mathrm{IR}} \\
(\mathrm{pc})\end{array}$ & $\begin{array}{l}\bar{T}_{\text {dust }} \\
(\mathrm{K})\end{array}$ & $\begin{array}{c}T_{\text {dust }}^{\text {peak }} \\
\text { (K) }\end{array}$ & $\begin{array}{c}M_{\text {tot }} \\
\left(10^{3} M_{\odot}\right)\end{array}$ & $\begin{array}{c}N_{\mathrm{H}_{2}} \\
\left(10^{22} \mathrm{~cm}^{-2}\right)\end{array}$ & $\begin{array}{c}n \\
\left(10^{3} \mathrm{~cm}^{3}\right) \\
\end{array}$ & $\begin{array}{l}\text { CO clump } \\
\text { counterpart }\end{array}$ \\
\hline IR1 & 181651.7 & -184135 & 14.7 & 0.57 & 1.2 & 33 & 62 & 2.4 & 2.4 & 5.1 & 1 \\
\hline IR2 & 181651.0 & -184308 & 3.6 & 0.21 & 1.0 & 27 & 34 & 0.8 & 1.1 & 3.1 & 2 \\
\hline IR3 & 181644.8 & -184019 & 7.2 & 0.23 & 1.2 & 25 & 29 & 1.7 & 1.4 & 3.4 & 4 \\
\hline
\end{tabular}

Planck function for a temperature $\bar{T}_{\text {dust }}($ Col. 7 in Table 3$)$, which was estimated by averaging the dust temperature map (see below) inside the area $A_{\mathrm{IR}}$ corresponding to each source.

The average volume density of each clump ( $n$, Col. 11 in Table 3) was derived, assuming a spherical geometry, as

$n=\frac{M_{\text {(tot) }}}{4 / 3 \pi R_{\mathrm{IR}}^{3} \mu m_{\mathrm{H}}}$,

where $m_{\mathrm{H}}$ is the mass of the hydrogen atom and $R_{\mathrm{IR}}(\mathrm{Col} .6$ in Table 3) is the effective radius of the structure, calculated as $R_{\mathrm{IR}}=\sqrt{A_{\mathrm{IR}} / \pi}$. The beam-averaged column density of the clumps $\left(N_{\mathrm{H}_{2}}\right.$, Col. 10 in Table 3$)$ was calculated using

$N_{\mathrm{H}_{2}}=R \frac{I_{870}}{\Omega_{\text {beam }} \kappa_{870} \mu m_{\mathrm{H}} B_{870}\left(\bar{T}_{\text {dust }}\right)}$

(Hildebrand 1983), where $I_{870}$ is the surface brightness emission at $870 \mu \mathrm{m}$ (Col. 5 in Table 3$)$ and $\Omega_{\text {beam }}$ is the beam solid angle $\left(\pi \theta_{\mathrm{HPBW}}^{2} / 4 \ln (2)\right)$.

The average and peak temperatures of the submillimeter sources, $\bar{T}_{\text {dust }}$ and $T_{\text {dust }}^{\text {peak }}$, were estimated using the inverse function of the ratio map of Herschel 250 and $350 \mu$ m images, i.e., $T_{\text {dust }}=f_{(T)}^{-1}$ (not shown here). Assuming a dust emissivity following a power law $\kappa_{v} \propto \nu^{\beta}$, which is $\beta$ the spectral index of the thermal dust emission, in the optically thin thermal dust emission regime $f_{(T)}$ has the parametric form

$f_{(T)}=\frac{S_{250}}{S_{350}}=\frac{B\left(250, T_{\text {dust }}\right)}{B\left(350, T_{\text {dust }}\right)}\left(\frac{250}{350}\right)^{\beta}$,

where $B\left(250, T_{\text {dust }}\right)$ and $B\left(350, T_{\text {dust }}\right)$ are the blackbody Planck function for a temperature $T_{\text {dust }}$ at the frequencies $250 \mu \mathrm{m}$ and $350 \mu \mathrm{m}$, respectively. The temperatures were calculated assuming a typical value $\beta=1$. The uncertainty in derived dust temperatures using this method was estimated to be about $\sim 10-15 \%$.

The significant higher temperature, derived particularly toward the region corresponding to clump 1, suggests the existence of powerful internal heating source/s that are very likely YSOs formed in the region and/or the stellar members of the cluster [BDS2003] 6 (see Sect. 6.2).

\section{Radio continuum emission}

The radio continuum emission distribution at $1.4 \mathrm{GHz}$ obtained from NVSS is shown in the upper panel of Fig. 4. Two bright sources, labeled as A and B, can be discerned projected onto the border of the IR nebula. The emission peak of source A is coincident with the center of the radio source G012.43-01.1112 3.7" $\times$ $3.1^{\prime \prime}$ in size detected at $5 \mathrm{GHz}$ by Urquhart et al. (2009), and is almost projected onto the emission peak of the CO clump 1 (see zoomed region in lower panel of Fig. 4). The total flux density derived for source A (see below) is in agreement with the value obtained by Beaumont \& Williams (2010) at the same frequency. From the flux density obtained by Urquhart et al. (2009)

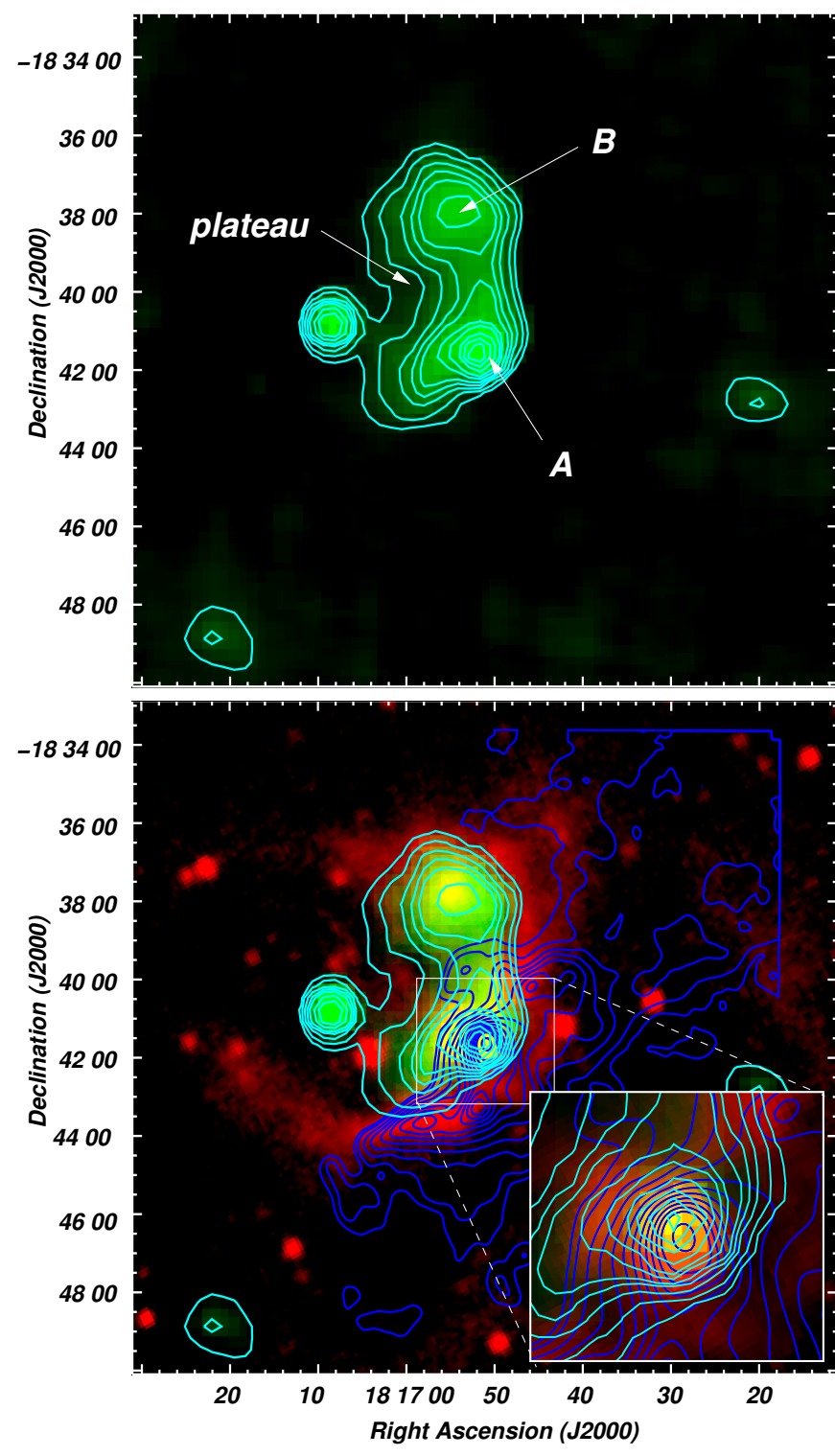

Fig. 4. Upper panel: radio continuum emission distribution at $1.4 \mathrm{GHz}$. Contour levels are 0.0022 ( $~ 5 \mathrm{rms}), 0.005,0.01,0.015,0.02,0.03,0.04$, $0.06,0.08$, and $0.1 \mathrm{mJy}$ beam $^{-1}$. Identified radio continuum sources are indicated (see text). Lower panel: overlay of the radio continuum emission at $1.4 \mathrm{GHz}$ (green color) on the MSX-A emission (red color) and $\mathrm{CO}$ emission in the velocity interval from 30 to $45 \mathrm{~km} \mathrm{~s}^{-1}$ (blue contours). A zoom of the central region is shown in the lower right corner.

$\left(S_{5 \mathrm{GHz}}=0.105 \mathrm{Jy}\right)$ and the flux density obtained in this work $\left(S_{1.4 \mathrm{GHz}}=0.09 \mathrm{Jy}\right)$, we derived a spectral index $\alpha=0.12 \pm 0.2$ $\left(S_{v} \propto v^{\alpha}\right)$; using the value derived by Beaumont $\&$ Williams (2010) $\left(S_{1.4 \mathrm{GHz}}=0.125 \mathrm{Jy}\right)$ we obtain $\alpha=-0.14 \pm 0.2$. Both values are compatible, within the errors, with the thermal free-free regime of an HII region. Then, source A is very likely associated 
Table 4. Properties derived for the radio continuum sources.

\begin{tabular}{cccccccc}
\hline \hline source & $\begin{array}{c}\text { RA } \\
(\mathrm{h} \mathrm{m} \mathrm{s})\end{array}$ & $\begin{array}{c}\text { Dec } \\
\left({ }^{\prime} \prime \prime\right)\end{array}$ & $\begin{array}{c}S_{1.4} \\
(\mathrm{Jy})\end{array}$ & $\begin{array}{c}n_{\mathrm{e}} \\
\left(\mathrm{cm}^{-3}\right)\end{array}$ & $\begin{array}{c}E M \\
\left(\mathrm{~cm}^{-6} \mathrm{pc}\right)\end{array}$ & $\begin{array}{c}M_{\text {ion }} \\
\left(M_{\odot}\right)\end{array}$ & $\begin{array}{c}N_{\mathrm{Lyc}} \\
\left(10^{47} \mathrm{~s}^{-1}\right)\end{array}$ \\
\hline $\mathrm{A}$ & 181651.4 & -184129 & 0.09 & 55 & 5000 & 4.6 & 1.0 \\
B & 181653.7 & -183753 & 0.03 & 30 & 1200 & 3.6 & 0.3 \\
plateau & - & - & 0.47 & - & - & - & 5.4 \\
\hline
\end{tabular}

with the ionization of the molecular gas in clump 1 by the action of stars possibly belonging to the stellar cluster [BDS2003]6 (see Sect. 6.2). Source B has a bright IR counterpart at $8 \mu \mathrm{m}$ (see Fig. 1) and is probably associated with IRAS 18139-1839. A third radio source at $1.4 \mathrm{GHz}$ is seen projected onto the bubble at RA, $\operatorname{Dec}(\mathrm{J} 2000)=18^{\mathrm{h}} 17^{\mathrm{m}} 08.4^{\mathrm{s}},-18^{\circ} 40^{\prime} 43^{\prime \prime}$. This source is very bright in the VLA MAGPIS image ${ }^{8}$ at $0.325 \mathrm{GHz}$ (not shown here). From its flux densities obtained at $0.325 \mathrm{GHz}$ and $1.4 \mathrm{GHz}$, we derived a nonthermal spectral index $\alpha=-1.9 \pm 1.7$, which indicates that this object is an extragalactic source.

A noteworthy feature is an extended horseshoe-shaped structure that connects sources A and B and can be detected almost toward the center of the bubble. The structure (hereafter the "plateau") shows a sharp border toward the west of the nebula (see the lower panel of Fig. 4), while it decreases smoothly toward the east. This indicates the existence of an electron density gradient and suggests that the structure is the radio continuum counterpart of the ionization front and ionized gas inside the nebula. The morphology of the plateau confirms the proposed scenario of an HII region bounded by ionization to the west (see Sects. 1 and 3.1). Neither Urquhart et al. (2009) nor Beaumont \& Williams (2010) reported this structure since their MAGPIS VLA images, obtained with more extended VLA configurations, probably were not able to detect extended emission.

In Table 4 we present some physical properties estimated for the ionized gas in A, B, and the plateau, assuming optically thin emission at $1.4 \mathrm{GHz}$. For the cases of $\mathrm{A}$ and $\mathrm{B}$, we use contour line limits of $0.027 \mathrm{mJy} \mathrm{beam}^{-1}(\sim 60 \mathrm{rms})$ and $0.021 \mathrm{mJy} \mathrm{beam}^{-1}(\sim 46 \mathrm{rms})$, respectively, to avoid the emission contributions from the plateau. Background-substracted flux densities derived for A and B and total flux density for the plateau (after substraction of A and B fluxes) are indicated in Col. 4 in Table 4.

Assuming spherical geometry for A and $\mathrm{B}$, and using the flux densities at $1.4 \mathrm{GHz}$, the electron density $\left(n_{\mathrm{e}}\right.$, Col. 5 in Table 4$)$ was derived using

$n_{\mathrm{e}}=3.113 \times 10^{2} \quad S_{1.4}^{0.5} T_{\mathrm{e}}^{0.25} d^{-0.5} b\left(v, T_{\mathrm{e}}\right)^{-0.5} \theta_{R}^{-1.5}$,

where $T_{\mathrm{e}}$ is the electron temperature (assumed to be $7 \times 10^{3} \mathrm{~K}$; Quireza et al. 2006), $S_{1.4}$ is the flux density at $1.4 \mathrm{GHz}, \theta_{\mathrm{R}}$ is the angular radius of the source, and $b\left(v, T_{\mathrm{e}}\right)=1+0.3195 \times$ $\log \left(T_{\mathrm{e}} / 10^{4} \mathrm{~K}\right)-0.213 \times \log (v / 1 \mathrm{GHz})$ (Panagia \& Walmsley 1978). The emission measure (EM, Col. 6 in Table 4) was obtained using

$E M=5.638 \times 10^{4} S_{1.4} T_{\mathrm{e}} b\left(v, T_{\mathrm{e}}\right) \theta_{R}^{-2}$.

The ionized gas mass $\left(M_{\text {ion }}\right.$, Col. 7 in Table 4$)$ was derived from

$M_{\text {ion }}=0.7934 S_{1.4}^{0.5} T_{\mathrm{e}}^{0.25} d^{2.5} b\left(v, T_{\mathrm{e}}\right)^{-0.5} \theta_{R}^{1.5}(1+Y)^{-1}$,

where $Y$ is the abundance ratio by number of $\mathrm{He}^{+}$to $\mathrm{H}^{+}$. The number of ionizing Lyman continuum photons $\left(N_{\mathrm{Lyc}} ; \mathrm{Col} .8\right.$ in

\footnotetext{
8 https://third.ucllnl.org/gps/index.html
}

Table 4) needed to sustain the ionization in the sources can be also calculated from the $1.4 \mathrm{GHz}$ emission using

$N_{\text {Lyc }}=7.58 \times 10^{48} T_{\mathrm{e}}^{-0.5} S_{1.4} d^{2}$

(Kurtz et al. 1994). The physical properties derived for the ionized gas in Table 4 should be considered lower limits owing to the missing flux of VLA.

Keeping in mind that values of $N_{\text {Lyc }}$ shown in Table 4 are lower limits since about $25-50 \%$ of the UV photons are absorbed by interstellar dust in the HII region (Inoue 2001), and taking into account particulary the value of $N_{\text {Lyc }}$ derived for the plateau, we estimate that the total amount of ionizing photons needed to sustain the current level of ionization in Sh2-39 is about $1.1 \times$ $10^{48} \mathrm{~s}^{-1}$. Adopting fluxes extracted from Sternberg et al. (2003), we estimate the spectral type of the ionizing star of Sh2-39 (not identified) to be about BOV. Alternatively, a handful of later Btype stars could also be responsible for powering the HII region.

\section{Star formation}

In this section we search for evidence of star formation in the region of Sh2-39. We use IR catalogs to identify candidate YSOs that are possibly associated with the nebula. We also pay special attention to the stellar cluster [BDS2003] 6 and its brightest member, projected at the border of the N5, which might have been formed by the action of the nebula on the surrounding gas.

\subsection{Identification of YSOs}

To search for candidate YSOs in N5, we analyzed the MSX, WISE, and Spitzer point source catalogs using the color-photometric criteria by Lumsden et al. (2002), Koenig \& Leisawitz 2014, and Allen et al. (2004), respectively. We searched for protostellar candidates within a region of $8^{\prime}$ in radius centered at the position RA, Dec $(\mathrm{J} 2000)=18^{\mathrm{h}} 16^{\mathrm{m}} 53.1^{\mathrm{s}}$, $-18^{\circ} 41^{\prime} 40^{\prime \prime}$. We took into consideration only sources projected onto the molecular emission, the IR nebula, and its interior. The coordinates of the identified candidate YSOs, names, and correlations with other catalogs are summarized in Table 5, while their locations are shown in Fig. 5. In Table 5 we also included the IRAS sources IRAS 18139-1842 and IRAS 18139-1839, which were classified as YSOs by Chan et al. (1996) and Codella et al. (1995). The source IRAS 18139-1842 was also identified as a candidate to be a site of an OB star (Wood \& Churchwell 1989).

The search for candidates in the MSX catalog allowed the identification of four sources classified as massive YSOs (MYSOs) or a compact HII region (CHII). The search in the Spitzer database allowed us to identify 4 Class I sources (protostars surrounded by dusty infalling envelopes) and 3 Class I/II sources (Class II sources: with emission dominated by accretion disks). We only took sources detected in the four Spitzer-IRAC bands into consideration. It should be kept in mind that only part of the nebula was surveyed with Spitzer. From 
N. U. Duronea et al.: Triggered massive star formation associated with the bubble HII region Sh2-39 (N5)

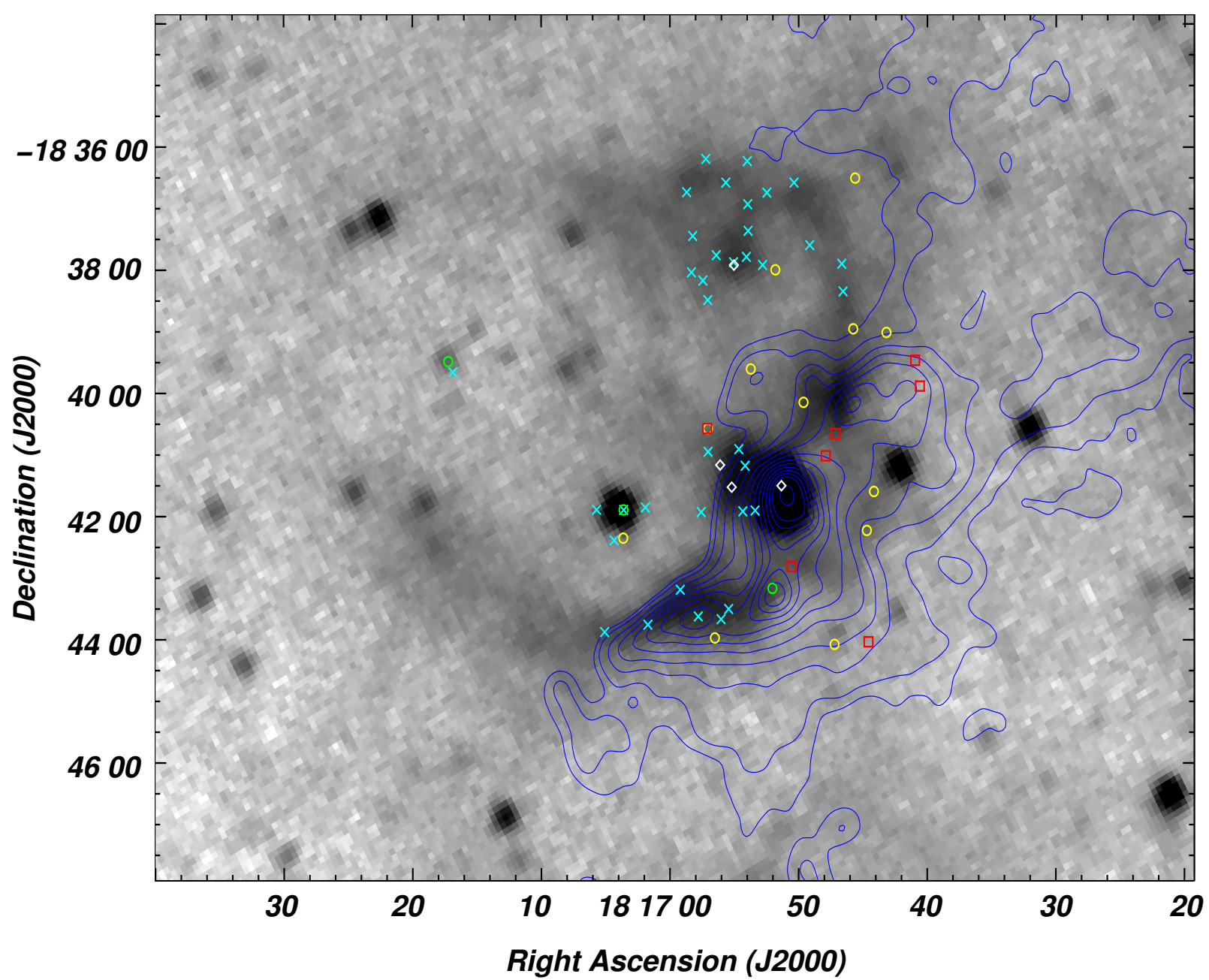

Fig. 5. Candidate YSOs projected onto the MSX image at $8.3 \mu \mathrm{m}$ (grayscale) and the CO emission (blue contours). The different symbols have the following meanings: white diamonds indicate MSX sources, red squares indicate Spitzer sources, green circles indicate WISE Class I sources, yellow circles indicate WISE Class II sources, and light blue crosses indicate Marton et al. sources.

the WISE database we identified 3 Class I and 12 Class II objects. For the sake of completeness, in Fig. 5 we include 35 ClassI/II candidates extracted from Marton et al. (2016), which were identified by the authors with the AllWISE catalog and the support vector machine (SVM) method.

The spatial distribution of the candidates YSOs shows that MSX sources 3, 4, and 6 (MYSO candidates), Spitzer source 11 (Class I/II), and a number of the Marton et al. WISE sources coincide with IRAS 18139-1842 (IRAS 1 in Table 5) and the CO clump 1. Radio recombination line observations ( $\mathrm{H} 87 \alpha$ and $\mathrm{H} 88 \alpha$ ) show emission toward this source at a velocity of $41 \mathrm{~km} \mathrm{~s}^{-1}$ (Lockman 1989), which indicates the existence of high density ionized gas at the same radial velocity as the CO. The stellar cluster [BDS2003] 6 is also projected in the region (see Fig. 6). Therefore, the observational evidence suggests that star formation is very active in the molecular clump 1 , which might explain the high peak dust temperature $(\sim 60 \mathrm{~K})$ derived from the IR continuum emission in this region (see Sect. 4).

Only Spitzer source 12 (Class I) and WISE source 14 (Class I) appear projected onto the CO emission of clump 2. WISE source 26 and a group of the Marton et al. WISE sources are projected onto CO clump 3, while Spitzer sources 7, 8, and 10, and WISE sources 17, 21, and 24 seem to be linked to CO clump 4. Additionally, Spitzer source 9, and WISE sources $18,19,22$, and 24 coincide with weak $\mathrm{CO}$ emission in the borders of the molecular region.

MSX source 5 (MYSO) and a number of Marton et al. WISE sources coincide with IRAS 2 (IRAS 18139-1839) and the radio continuum source $\mathrm{B}$, indicating that star formation is also active in this region. However, the lack of discernible $\mathrm{CO}$ emission within the velocity range linked to the bubble casts doubts on the association of this star forming spot with the HII region.

Finally, a group of WISE and Marton et al. sources is present around RA, Dec $(\mathrm{J} 2000)=18^{\mathrm{h}} 17^{\mathrm{m}} 04.1^{\mathrm{s}},-18^{\circ} 41^{\prime} 57^{\prime \prime}$, almost projected onto a bright MSX source. The relation of these sources to the IR bubble is not clear with the present data.

Hence, the presence of many candidate YSOs projected onto the molecular cloud that encircles the western and southwestern rims of $\mathrm{N} 5$ confirms that active star formation has developed in the borders of the bubble, particulary in the region of clump 1, which is a very active star forming spot.

\subsection{Star cluster [BDS2003] 6 and 2MASS J18165113-1841488}

As mentioned in Sect. 1, Bica et al. (2003) identified a small star cluster candidate, with nomenclature [BDS2003] 6 in their catalog, in the region of Sh2-39. Later, Morales et al. (2013) estimated a distance of $4.15 \mathrm{kpc}$ to the cluster. As previously 
Table 5. Identified candidate YSOs projected onto N 5.

\begin{tabular}{|c|c|c|c|c|}
\hline $\begin{array}{c}\text { IRAS sources } \\
\#\end{array}$ & $\begin{array}{c}\text { RA,Dec } \\
\left(\mathrm{h} \mathrm{m} \mathrm{s},{ }^{\circ \prime \prime \prime}\right)\end{array}$ & Catalog name & Class & \\
\hline 1 & $181653.1,-184140$ & IRAS 18139-1842 & YSO & \\
\hline 2 & $181653.2,-183757$ & IRAS 18139-1839 & YSO & \\
\hline $\begin{array}{c}\text { MSX sources } \\
\#\end{array}$ & & & & $\begin{array}{c}\text { Matching with } \\
\text { other sources }\end{array}$ \\
\hline 3 & $181656.1,-184109$ & G012.4452-01.1255 & MYSO/CHII & \\
\hline 4 & $181655.2,-184132$ & G012.4382-01.1252 & MYSO & \\
\hline 5 & $181655.0,-183755$ & G012.4909-01.0962 & MYSO & $c$ \\
\hline 6 & $181651.3,-184130$ & G012.4314-01.1117 & MYSO & \\
\hline \multicolumn{5}{|l|}{$\begin{array}{c}\text { Spitzer sources } \\
\#\end{array}$} \\
\hline 7 & $181640.583,-183953.02$ & G012.4350-01.0616 & I & \\
\hline 8 & $181640.954,-183927.94$ & G012.4418-01.0596 & I & $a$ \\
\hline 9 & $181644.566,-184402.22$ & G012.3816-01.1083 & I & $a$ \\
\hline 10 & $181647.132,-184039.39$ & G012.4360-01.0905 & $\mathrm{I} / \mathrm{II}$ & \\
\hline 11 & $181647.887,-184101.02$ & G012.4321-01.0959 & $\mathrm{I} / \mathrm{II}$ & \\
\hline 12 & $181650.554,-184248.80$ & G012.4107-01.1194 & $\mathrm{I}$ & \\
\hline 13 & $181657.106,-184034.36$ & G012.4559-01.1244 & $\mathrm{I} / \mathrm{II}$ & 27 \\
\hline \multicolumn{5}{|l|}{$\begin{array}{c}\text { WISE sources } \\
\ldots\end{array}$} \\
\hline 14 & $181652.045,-184310.16$ & J181652.04-184310.1 & I & $b$ \\
\hline 15 & $181703.605,-184153.73$ & J181703.60-184153.7 & I & $c$ \\
\hline 16 & $181717.233,-183929.28$ & J181717.23-183929.2 & I & $c$ \\
\hline 17 & $181643.158,-183901.24$ & $\mathrm{~J} 181643.15-183901.2$ & II & $b$ \\
\hline 18 & $181644.147,-184135.80$ & J181644.14-184135.8 & II & $b$ \\
\hline 19 & $181644.702,-184213.71$ & J181644.70-184213.7 & II & $b$ \\
\hline 20 & $181645.609,-183630.91$ & J181645.60-183630.9 & II & $b$ \\
\hline 21 & $181645.762,-183857.27$ & J181645.76-183857.2 & II & $b$ \\
\hline 22 & $181647.073,-184403.27$ & J181647.07-184403.2 & II & \\
\hline 23 & $181649.622,-184008.44$ & J181649.62-184008.4 & II & $b$ \\
\hline 24 & $181651.811,-183757.27$ & $\mathrm{~J} 181651.81-183757.2$ & II & $b$ \\
\hline 25 & $181653.809,-183936.41$ & J181653.80-183937.4 & II & $b$ \\
\hline 26 & $181656.510,-184358.57$ & J181656.50-184358.4 & II & \\
\hline 27 & $181657.048,-184034.64$ & J181657.04-184034.6 & II & 13 \\
\hline 28 & $181703.605,-184153.73$ & J181703.60-184153.7 & II & \\
\hline
\end{tabular}

Notes. Panel $a$ : coincidence with WISE source not catalogued as YSO. Panel b: coincidence with Spitzer source not catalogued as YSO. Panel $c$ : Identified by Marton et al. (2016) as YSO Class II.

mentioned, the cluster is projected onto the region of $\mathrm{CO}$ clump 1 , which is a very active center of star formation.

The color $(J, H, K 1)$ image of $\sim 2 ! 3 \times 2 ! 3$ around the center of [BDS2003] 6 retrieved from UKIDSS is shown in the upper panel of Fig. 6. By an aperture photometry performed on these bands, only a handful of stars of a poorly populated mainsequence (MS) and pre-main-sequence (PMS) were identified in the color-color and color-magnitude diagrams as probable members of the cluster. They have magnitudes in the range 10$16 \mathrm{mag}$ in $\mathrm{Ks}$ and colors in the range $1.3<(J-K \mathrm{~s})<4.5$. No further analysis was performed on these data owing to the small number of stars in the UKIDSS photometric catalog.

In the lower panel of Fig. 6 we show the $K$ s band image of [BDS2003] 6 obtained from the VVVX survey. We performed a PSF photometry transforming magnitudes to the 2MASS system using common stars (see Borissova et al. 2011, 2014, for details of the procedure). The photometry was used to construct the stellar surface-density map of the cluster and to determine its visual radius. We performed direct star counting with a $10 \operatorname{arcsec}$ space radius, assuming spherical symmetry. That number is then divided by the area of the rings to determine the stellar density. The obtained spatial distribution map of the stellar surface density is shown in the upper and middle panels of Fig. 7. The density peak is about 10 times larger than the surface density of the comparison field (estimated to be $0.025 \pm 0.009 \mathrm{stars} / \mathrm{arcmin}^{2}$ ), which confirms the clustering nature of [BDS2003] 6. To estimate the size of the cluster, we used the projected star surface density as a function of the radius (lower panel of Fig. 7) and the theoretical profile of Elson et al. (1987). We obtained a visual radius of $44^{\prime \prime} \pm 8^{\prime \prime}(0.87 \mathrm{pc}$ at a distance of $4.1 \mathrm{kpc})$, which is almost twice the radius estimated by Morales et al. (2013) and approximately the same size of clump 1. A quantitative estimate of the cluster compactness can be obtained from the density-contrast parameter, $\delta$, proposed by Bonatto \& Bica (2009), which connects the surface density of the cluster, $\sigma_{\mathrm{cl}}$, with the residual background density, $\sigma_{\mathrm{bg}}$, by $\delta=\sigma_{\mathrm{cl}} / \sigma_{\mathrm{bg}}+1$. For the case of [BDS2003] 6 we estimated $\delta=11.8$, which represents a relatively compact cluster.

To estimate the reddening and distance to the cluster, we used the spectroscopic parallax of its brightest source, 


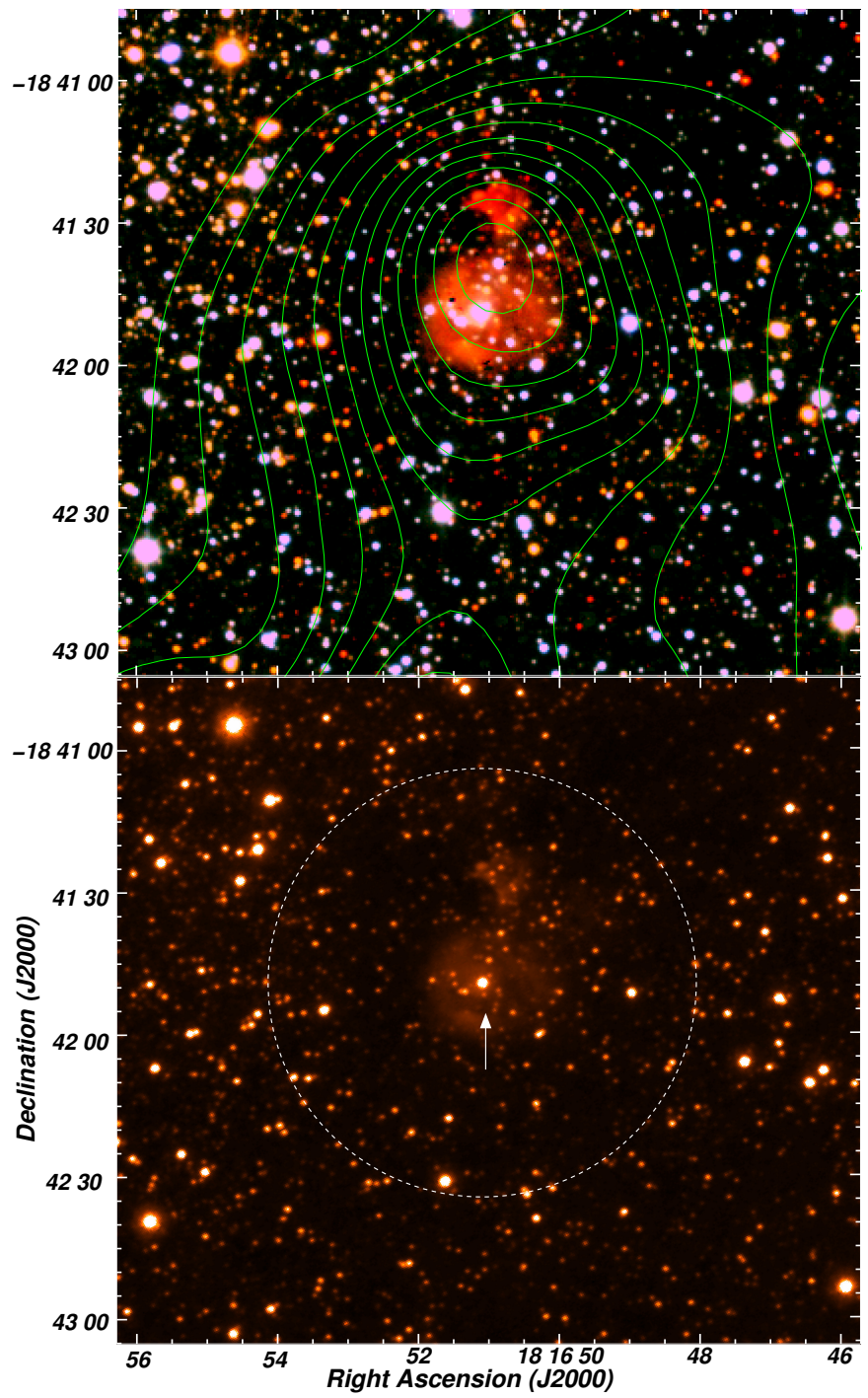

Fig. 6. Upper panel: color $(J, H, K 1)$ image of $\sim 2 ! 3 \times 2 ! 3$ around the center of [BDS2003] 6 retrieved from the UKIDSSDR10PLUS. Green contours indicate the $\mathrm{CO}$ emission of clump 1. Lower panel: Ks band image of [BDS2003] 6 retrieved from the VVVX survey is shown. The radius derived for the cluster (see text) is indicated with the white dotted circle. The location of Obj1 is indicated by the white arrow.

2MASSJ18165113-1841488 (hereafter Obj1), observed with ARCoIRIS. The position of this source is indicated in the lower panel of Fig. 6. Spectral classification was performed with atlases of infrared spectra that feature spectral types stemming from optical studies (e.g., Hanson et al. 2005). The reduced spectrum is shown in Fig. 8, where He I, Paschen, and Brackett lines in absorption are clearly visible, but no He II is observed. In addition, we took into consideration the position of Obj1 on the color-magnitude diagram (not shown here) to discern its luminosity class. Gathering all this information, we derived a spectral type $08-9 \mathrm{Ve}$. It is worth mentioning that the profiles in the lines $\mathrm{P} \beta, \mathrm{Br} 23$, and $\mathrm{Br} \gamma$ are partly filled (see lower panel of Fig. 8), which is believed to be caused by an enhanced mass-loss episode, such as stellar winds or molecular outflows (Bik et al. 2008). The presence of a very bright shell-like nebulosity around Obj1 (see upper and lower panels of Fig. 6) provides strong support for this scenario. A second nebulosity is seen $\sim 25^{\prime \prime}$ north of Obj1 at RA, Dec $(\mathrm{J} 2000)=18^{\mathrm{h}} 16^{\mathrm{m}} 50.8^{\mathrm{s}},-18^{\circ} 41^{\prime} 27^{\prime \prime}$, having the same size and position as the radio source G012.4317-01.1112
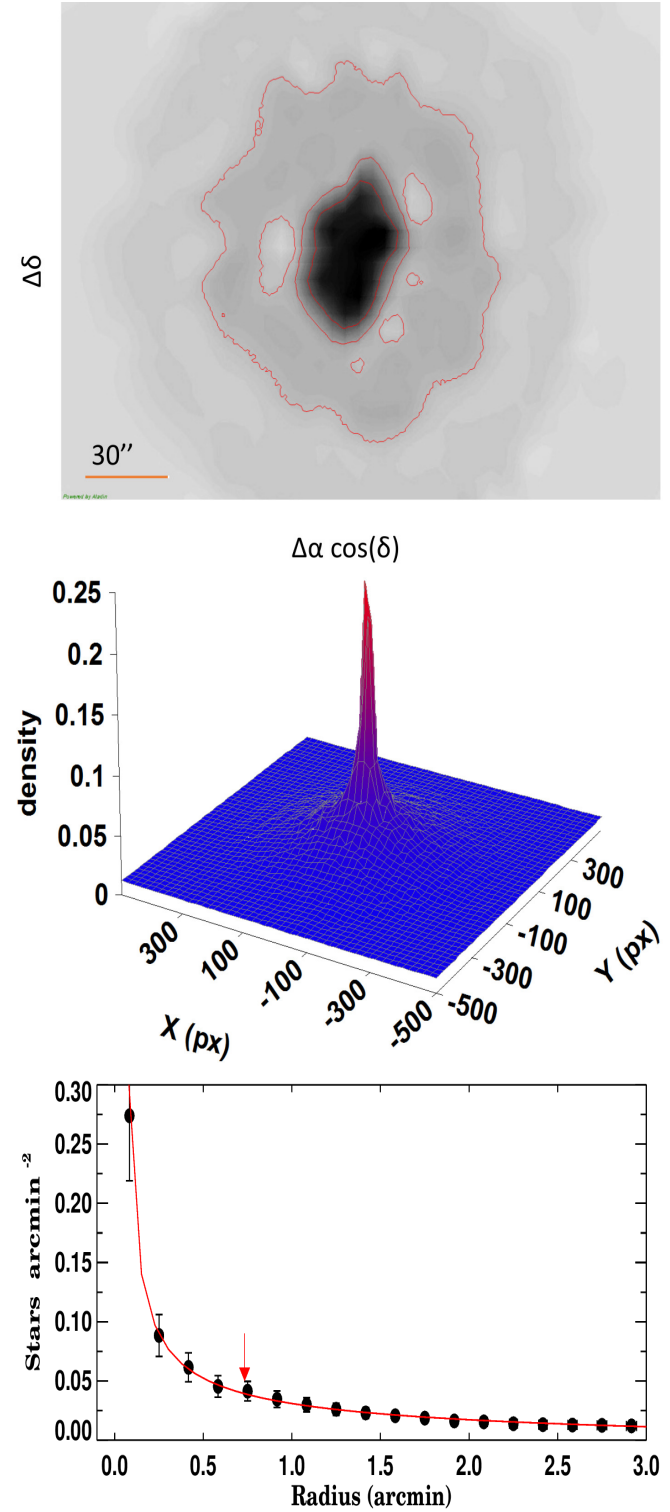

Fig. 7. Upper panel: two-dimensional stellar surface-density map obtained in a region $3^{\prime}$ around the center of [BDS2003] 6. The red lines correspond to the contours representing 3, 5 , and 10 sigma of the background surface density. Middle panel: a 3D stellar surface-density map is shown. Lower panel: radial density profile of the cluster is shown. The solid line stands for the best fit and the arrow indicates the radius derived for the cluster.

reported by Urquhart et al. (2009). This coincidence suggests that this nebulosity could be the IR counterpart of radio source A. A higher spatial resolution study of these nebulosities might provide important information about the characteristics of Obj1 and the rest of the stellar content of the cluster.

Individual extinction and distance were estimated using the spectral classification of Obj 1 and the intrinsic colors and luminosities cited by Martins \& Plez (2006) for O-type stars. The uncertainties are calculated by quadratically adding the uncertainties of the photometry and the spectral classification (e.g., two subtypes). We derived $E(J-K)=1.6 \pm 0.3$ and $(M-m)_{0}=$ $13.1 \pm 0.43$, which corresponds to a distance $d=4.2 \pm 0.8 \mathrm{kpc}$. This value is in excellent agreement with the distance estimated by Morales et al. (2013) and the kinematical distance adopted for Sh2-39. This confirms undoubtedly that Obj1 and the stellar 

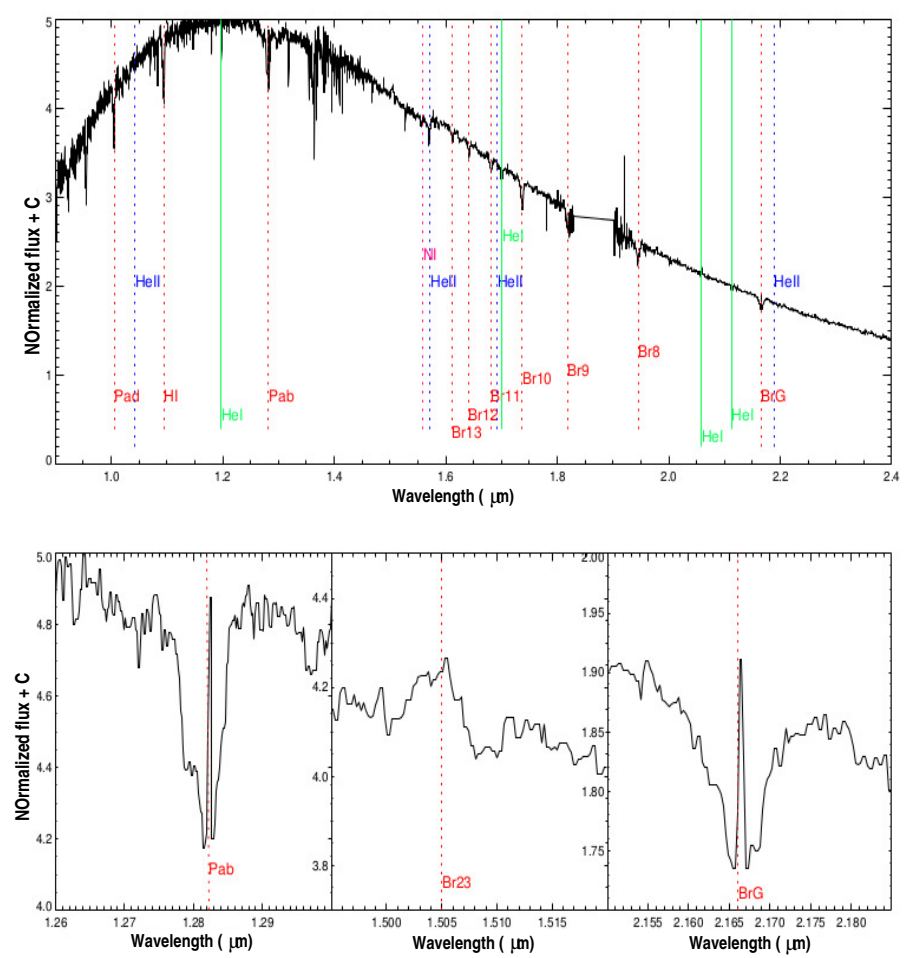

Fig. 8. ARCoIRIS spectrum of Obj1 (2MASSJ18165113-1841488) The observed spectral lines are indicated. Lines with emission components are zoomed.

cluster [BDS2003] 6 are physically associated with N5. The high dust temperatures derived in the region of clump 1 (see Sect. 4) could be explained by the presence of the cluster that is still embedded in the the dense molecular gas of the clump. The formation of the cluster was very likely triggered by the expansion of the ionization front over its parental molecular cloud. A discussion of this scenario is presented in Sect. 7.3.

\section{Discussion}

\subsection{Stability of the clumps}

Classical virial equilibrium analysis establishes that for a stable, self-gravitating spherical mass, the kinetic energy must equal half of the potential energy. Then, the virial mass estimated with Eq. (7) is the minimum mass required in order for a clump to be gravitationally bound. This means that if $M_{\mathrm{vir}}$ is smaller than the mass estimated with any other technique, the clump is gravitationally unstable and has the potential to collapse to form new stars. In the same way, if $M_{\text {vir }}$ is larger, the clump has too much kinetic energy and is unable to collapse.

Considering the range of molecular masses detailed in Tables 2 and 3 for clump 1, we estimate the mass ratio ranges $M_{\text {int }} / M_{\text {vir }}=0.9-1.5$ and $M_{\mathrm{LTE}} / M_{\mathrm{vir}}=0.6-2.5$, which suggests a more likely gravitational collapse. For the case of the ratio $M_{\mathrm{tot}} / M_{\mathrm{vir}}=1.1-1.8$ the result is more conclusive. As seen before, clump 1 seems to be a very active massive star forming clump, which confirms that the clump is, or else was, undergoing gravitational collapse. For the case of clump 2, where two candidate YSOs were found projected onto it, the ratios are $M_{\text {int }} / M_{\text {vir }}=2.4-3.6$ and $M_{\mathrm{LTE}} / M_{\mathrm{vir}}=0.6-2.5$, and $M_{\mathrm{tot}} / M_{\mathrm{vir}}=$ $0.9-1.3$, which also suggests a likely gravitational collapse.

The mass-radius relationship of nearby molecular clouds was investigated in two sequent papers by Kauffmann et al. (2010a) and Kauffmann et al. (2010b), who found that clouds that were devoid of any high-mass star formation usually obeyed the empirical relationship $m(r) \leq 870 M_{\odot} \times\left(R_{\text {eff }} / \mathrm{pc}\right)^{1.33}$. Afterward, Urquhart et al. (2013) studied 577 submillimeter continuum clouds and determined a lower surface density of $0.05 \mathrm{gr} / \mathrm{cm}^{-2}$ $\left(M>750 M_{\odot} \times\left(R_{\mathrm{eff}} / \mathrm{pc}\right)^{2}\right)$ as a reliable empirical lower bound for the clump surface density required for massive star formation. Using $R_{\text {eff }}$ derived for clump 1 (see Table 2) we obtain masses of $1.3 \times 10^{3} M_{\odot}$ and $1.5 \times 10^{3} M_{\odot}$ for the Kauffmann et al. and Urquhart et al. relations, exceeding both mass limit conditions for the massive star formation. For the case of clump 2 we obtain masses of $2.0 \times 10^{3} M_{\odot}$ and $2.7 \times 10^{3} M_{\odot}$, exceeding only the mass limit of Kaufmann et al.

Since no $\mathrm{HCO}^{+}$emission could be observed for clumps 3 and 4 , it was not possible to perform a virial analysis. Effective radius estimated for these clumps indicate that both clumps have masses in the limit of the Kauffmann et al. masses, but are under the Urquhart et al. limit. As seen in Sect. 6.1, star formation activity seems to be very active in clump 3, which may indicate that a gravitational collapse may have also occurred here.

\subsection{Expansion of the nebula}

In previous sections, we show observational evidence suggesting that the ionized nebula is expanding against the molecular cloud. Although no conclusive evidence of expansion can be obtained from molecular data, the dynamical time, $t_{\mathrm{dyn}}$, and the expansion velocity, $\dot{R}_{\text {HII }}$, can be obtained from the radio continuum emission with the model of Dyson \& Williams (1997). The radius of an HII region $\left(R_{\mathrm{HII}}\right)$ in a uniform medium is given by

$\frac{R_{\mathrm{HII}}}{R_{\mathrm{S}}}=\left(1+\frac{7 v_{\mathrm{s}} t_{\mathrm{dyn}}}{4 R_{\mathrm{S}}}\right)^{4 / 7}$,

where $v_{\mathrm{s}}$ is the sound speed in the ionized gas (assumed to be $\sim 10 \mathrm{~km} \mathrm{~s}^{-1}$ ), and $R_{\mathrm{S}}$ is the radius of the Strömgren sphere (Strömgren 1939) before expanding, given by

$R_{\mathrm{S}}=\left(\frac{3 N_{\mathrm{Lyc}}^{*}}{4 \pi\left(n_{0}\right)^{2} \alpha_{\beta}}\right)^{1 / 3}$,

where $n_{0}$ is the original ambient density, and $\alpha_{\beta}$ is the hydrogen recombination coefficient to all levels above the ground level (adopted as $3.5 \times 10^{-13}$ for $T_{\mathrm{e}}=7000$; Lequeux 2005). To estimate $n_{0}$ we averaged the total mass of the molecular clumps $\left(\sim 7.5 \times 10^{3} M_{\odot}\right)$ over a sphere $4.8 \mathrm{pc}$ in radius $\left(\sim 4^{\prime}\right.$ at a distance of $4.1 \mathrm{kpc}$ ), which yields $n_{0}=620 \mathrm{~cm}^{-3}$. Adopting $N_{\text {Lyc }}^{*}=1.1 \times 10^{48} \mathrm{~s}^{-1}$ (see Sect. 5), we infer $t_{\text {dyn }} \approx 8.4 \times 10^{6} \mathrm{yr}$. We keep in mind that when considering only the mass of the molecular clumps and neglecting extended molecular emission, a considerable amount of molecular mass have not been taken into account, which yields a lower limit for $n_{0}$. Therefore, $t_{\text {dyn }}$ could be even larger than the value derived above.

The expansion velocity of the HII region can be estimated by deriving Eq. (16) in time

$\dot{R}_{\mathrm{HII}}=v_{\mathrm{s}}\left(\frac{R_{\mathrm{HII}}}{R_{\mathrm{s}}}\right)^{-3 / 4}$,

yielding an expansion velocity of about $\sim 0.4 \mathrm{~km} \mathrm{~s}^{-1}$.

The obtained dynamical age and expansion velocity indicates that Sh2-39 is older and slower compared with typical Galactic bubble HII regions (e.g., Gum 31, Cappa et al. 2008; 
Sh2-173, Cichowolski et al. 2009; G8.14+0.23, Dewangan et al. 2012; RCW 120, Anderson et al. 2015).

In Sect. 5 we estimated the spectral type of the ionizing star/s in Sh2-39 to be BOV or later. The total main-sequence lifetime for these spectral types is estimated to be $\sim 10^{7} \mathrm{yr}$ (Hansen \& Kawaler 1994), which is in agreement with the dynamical time derived for Sh2-39.

\subsection{Triggered star formation scenario}

To test whether the $\mathrm{C} \& \mathrm{C}$ mechanism is responsible for the formation of [BDS2003] 6 and the candidate YSOs identified in the periphery of Sh2-39, we compared the dynamical age of the HII region, $t_{\mathrm{dyn}}$, with the fragmentation time of the collected molecular gas, $t_{\text {frag }}$, predicted with the model by Whitworth et al. (1994).

To estimate $t_{\text {frag }}$, and the size of the HII region at the fragmentation time $\left(R_{\text {frag }}\right)$, we use

$t_{\text {frag }}\left[10^{6} \mathrm{yr}\right]=1.56 \mathrm{a}_{0.2}^{4 / 11} \mathrm{n}_{3}^{-6 / 11} \mathrm{~N}_{49}^{-1 / 11}\left[10^{6} \mathrm{yr}\right]$

$R_{\text {frag }}[\mathrm{pc}]=5.8 \mathrm{a}_{0.2}^{4 / 11} \mathrm{n}_{3}^{-6 / 11} \mathrm{~N}_{49}^{1 / 11}[\mathrm{pc}]$,

where $a_{0.2}$ is the isothermal sound speed in the compressed layer, in units of $0.2 \mathrm{~km} \mathrm{~s}^{-1}\left(a_{\mathrm{s}} / 0.2 \mathrm{~km} \mathrm{~s}^{-1}\right) ; n_{3}$ is the mean density of the surrounding homogeneous infinite medium into which the HII region expands, in units of $1000 \mathrm{~cm}^{-3}\left(n_{0} / 1000 \mathrm{~cm}^{-3}\right)$; and $N_{49}$ is the number of ionizing photons, in units of $10^{49}\left(N_{49}=\right.$ $\left.N_{\text {Lyc }}^{*} / 10^{49} \mathrm{~s}^{-1}\right)$. As pointed out in Whitworth et al. (1994), $a_{\mathrm{s}}=$ $0.2 \mathrm{~km} \mathrm{~s}^{-1}$ is likely a lower limit for the sound of speed in the collected layer, since both turbulence and extra heating from intense sub-Lyman-continuum photons leaking from the HII region could increase this value. Therefore, in the following we use the typically adopted range $a_{\mathrm{s}}=0.2-0.6 \mathrm{~km} \mathrm{~s}^{-1}$ for the collected layer (e.g., Dewangan et al. 2012). We obtained a fragmentation time range $t_{\text {frag }}=2 .-3.6 \times 10^{6} \mathrm{yr}$ and a frangmentation radius range $R_{\text {frag }}=6.2-9.3$ pc. A direct comparison between $t_{\text {dyn }}$ and $t_{\text {frag }}$ suggests that $\mathrm{C} \& \mathrm{C}$ is a very plausible scenario for the star formation in the border of N5. However, a comparison between $R_{\mathrm{HII}}$ and $R_{\text {frag }}$ suggests that the fragmentation should have occurred some later. A combined C\&C/RDI mechanism scenario is also possible. More observational data are necessary to shed some light on these issues.

We keep in mind that some discrepancies are always expected between model and observations, namely: a) the model of Whitworth et al. (1994) assumes spherical symmetry around the central star and expansion into a homogeneous infinite medium. This is certainly not the case of Sh2-39, which appears to have been formed at the edge of its parental cloud (blyster-type HII region); b) if the fragmentation had symmetry around the center of Sh2-39, more candidate YSOs should be detected in the direction of the ionized gas; c) although the ring morphology in the IR emission is a common feature in many Galactic HII regions (e.g., Watson et al. 2008; Deharveng et al. 2009; Cappa et al. 2016), this has initiated some debate on whether these structures are flat (few parsecs) two-dimensional ring-like objects (Beaumont \& Williams 2010; Arce et al. 2011) or projected three-dimensional bubbles (Deharveng et al. 2010; Anderson et al. 2012, 2015; Duronea et al. 2015).

\section{Concluding remarks}

As part of a project aimed to study the physical characteristics of Galactic IR bubbles and to explore their influence in triggering massive star formation, we present a multiwavelength analysis of the northern HII region bubble Sh2-39 (N5) and its environs. We used ASTE CO(3-2) and $\mathrm{HCO}^{+}(4-3)$ line data to explore the characteristics of the molecular cloud associated with the nebula and to study the physical properties of the star forming locations. The characteristics of the dust and ionized gas were explored with archival data, while a stellar source member of the cluster [BDS2003] 6, projected onto the border of the IR bubble, was studied with new IR data.

Using the $\mathrm{CO}(3-2)$ line data, we identified a molecular cloud concentrated between $\sim 30 \mathrm{~km} \mathrm{~s}^{-1}$ and $46 \mathrm{~km} \mathrm{~s}^{-1}$ toward the western and southern borders of the IR nebula, coincident with the PAH emission detected at $8.28 \mu \mathrm{m}$, indicating that Sh2-39 is a blister-type HII region bounded by ionization to the west and density to the east. We identified four molecular clumps with radii between 1.4 and $1.9 \mathrm{pc}$, integrated masses between $1.4 \times 10^{3}$ and $2.2 \times 10^{3} M_{\odot}$, and volume densities between $1.5 \times 10^{3}$ and $3.3 \times 10^{3} \mathrm{~cm}^{-3}$. The clumps are located over the western border of the IR bubble and were likely formed by the action of the HII region expanding against its parental molecular cloud. Clumps 1 and 2 have counterparts in the $\mathrm{HCO}^{+}(4-3)$ line and in the $870 \mu \mathrm{m}$ dust continuum emission, while clump 3 only in the $870 \mu \mathrm{m}$ emission. This indicates that these clumps have high densities and are good sites to look for massive star formation. A virial analysis performed for clumps 1 and 2 indicates that both clumps are gravitationally unstable and have the potential to collapse to form new stars.

Using IR point source catalogs, we identified a number of candidate YSOs projected onto the molecular cloud next to N5 confirming that active star formation has developed in the borders of the bubble. The location of the stellar cluster [BDS2003] 6, projected onto the molecular clump 1, is also indicative of the star formation activity. An analysis performed with new available IR data indicates that the cluster is $0.87 \mathrm{pc}$ in radius and confirms its clustering nature. A spectroscopic study of the brightest member of [BDS2003] 6 (Obj1) reveals a O8-9V star. The estimated the distance to the cluster, $4.2 \pm 0.8 \mathrm{kpc}$, is in excellent agreement with previous distance estimations for Sh239. This certainly confirms the association of the cluster with the nebula and gives more support to the massive star formation scenario on the edge of the HII region, particulary on clump 1.

The analysis of the NVSS radio continuum emission distribution at $1.4 \mathrm{GHz}$ allowed us to identify three noteworthy features projected toward the center and border of the IR bubble. The morphology of the more extended feature (plateau) perfectly matches the inner border of the PDR, which indicates that this structure is the radio counterpart of the ionization front and the HII region. We estimate a single B0V, or else a handful of later B-type stars to be responsible for powering the HII region. We derived a dynamical age for the nebula of $\sim 8.4 \times 10^{6} \mathrm{yr}$, which is in agreement with the main-sequence lifetime of a B0V star or later. The dynamical age and expansion velocity derived for Sh2-39 indicates that the bubble is older and slower compared with typical Galactic bubble HII regions. The other two radio sources (A and B) were probably formed during the expansion of Sh2-39.

After comparing the dynamical age of the HII region and the fragmentation time of the molecular gas around the HII region, we concluded that the $\mathrm{C} \& \mathrm{C}$ process may indeed be important in the collected layers of gas at the edge of the bubble, boosting the formation of the candidate YSOs detected in the molecular gas around Sh2-39 and the cluster [BDS2003] 6. However, a comparison between the observed radius of the bubble with 
the fragmentation radius suggests that the fragmentation should have occurred later.

Acknowledgements. We acknowledge the anonymous referee for her/his help ful comments and suggestions that improved the presentation of this paper. This project was partially financed by CONICET of Argentina under projects PIP 00356, and PIP00107, and from UNLP, 2012-2014 PPID/G002 and 11/G120. L.B. acknowledges support by CONICYT project PFB-06. Support for J.B. is provided by the Ministry of Economy, Development, and Tourism's Millennium Science Initiative through grant IC120009, awarded to The Millennium Institute of Astrophysics, MAS. M.A.K. was supported by a Chilean FONDECYT fellowship grant (No. 3150319) from the Comisión Nacional de Investigación Científica y Tecnológica (CONICYT) and the Ministry of Economy, Development, and Tourism's Millennium Science Initiative through grant IC120009, awarded to The Millennium Institute of Astrophysics.

\section{References}

Allen, L. E., Calvet, N., D’Alessio, P., et al. 2004, ApJS, 154, 363 Anderson, L. D., Zavagno, A., Deharveng, L., et al. 2012, A\&A, 542, A10 Anderson, L. D., Deharveng, L., Zavagno, A., et al. 2015, ApJ, 800, 101

Arce, H. G., Borkin, M. A., Goodman, A. A., Pineda, J. E., \& Beaumont, C. N 2011, ApJ, 742, 105

Beaumont, C. N., \& Williams, J. P. 2010, ApJ, 709, 791

Bica, E., Dutra, C. M., Soares, J., \& Barbuy, B. 2003, A\&A, 404, 223

Bik, A., Kaper, L., Hanson, M. M., \& Waters, L. B. F. M. 2008, in Mass Loss from Stars and the Evolution of Stellar Clusters, eds. A. de Koter, L. J. Smith, \& L. B. F. M. Waters, ASP Conf. Ser., 388, 143

Blitz, L. 1993, in Protostars and Planets III, eds. E. H. Levy, \& J. I. Lunine, 125

Bonatto, C., \& Bica, E. 2009, MNRAS, 394, 2127

Borissova, J., Bonatto, C., Kurtev, R., et al. 2011, A\&A, 532, A131

Borissova, J., Chené, A.-N., Ramírez Alegría, S., et al. 2014, A\&A, 569, A24

Brand, J., Massi, F., Zavagno, A., Deharveng, L., \& Lefloch, B. 2011, A\&A, 527 A62

Bronfman, L., Nyman, L.-A., \& May, J. 1996, A\&AS, 115, 81

Cappa, C., Niemela, V. S., Amorín, R., \& Vasquez, J. 2008, A\&A, 477, 173

Cappa, C. E., Duronea, N., Firpo, V., et al. 2016, A\&A, 585, A30

Chan, S. J., Henning, T., \& Schreyer, K. 1996, A\&AS, 115, 285

Churchwell, E., Povich, M. S., Allen, D., et al. 2006, ApJ, 649, 759

Cichowolski, S., Romero, G. A., Ortega, M. E., Cappa, C. E., \& Vasquez, J 2009, MNRAS, 394, 900

Codella, C., Palumbo, G. G. C., Pareschi, G., et al. 1995, MNRAS, 276, 57

Condon, J. J., Cotton, W. D., Greisen, E. W., et al. 1998, AJ, 115, 1693

Cortes, P. C. 2011, ApJ, 743, 194

Cortes, P. C., Parra, R., Cortes, J. R., \& Hardy, E. 2010, A\&A, 519, A35

Cushing, M. C., Vacca, W. D., \& Rayner, J. T. 2004, PASP, 116, 362

Deharveng, L., Lefloch, B., Zavagno, A., et al. 2003, A\&A, 408, L25

Deharveng, L., Lefloch, B., Kurtz, S., et al. 2008, A\&A, 482, 585

Deharveng, L., Zavagno, A., Schuller, F., et al. 2009, A\&A, 496, 177

Deharveng, L., Schuller, F., Anderson, L. D., et al. 2010, A\&A, 523, A6

Dewangan, L. K., Ojha, D. K., Anandarao, B. G., Ghosh, S. K., \& Chakraborti, S. 2012, ApJ, 756, 151

Draine, B. T., Dale, D. A., Bendo, G., et al. 2007, ApJ, 663, 866

Duronea, N. U., Vasquez, J., Gómez, L., et al. 2015, A\&A, 582, A2

Dyson, J. E., \& Williams, D. A. 1997, The physics of the interstellar medium, eds. J. E., Dyson, \& D. A. Williams (Bristol: Institute of Physics Publishing)

Elmegreen, B. G., \& Lada, C. J. 1977, ApJ, 214, 725

Elson, R. A. W., Fall, S. M., \& Freeman, K. C. 1987, ApJ, 323, 54

Ezawa, H., Kawabe, R., Kohno, K., \& Yamamoto, S. 2004, in Ground-based Telescopes, ed. J. M. Oschmann, Jr., Proc. SPIE, 5489, 763

Ezawa, H., Kohno, K., Kawabe, R., et al. 2008, in Ground-based and Airborne Telescopes II, Proc. SPIE, 7012, 701208
Faúndez, S., Bronfman, L., Garay, G., et al. 2004, A\&A, 426, 97 Fazio, G. G., Hora, J. L., Allen, L. E., et al. 2004, ApJS, 154, 10 Griffin, M. J., Abergel, A., Abreu, A., et al. 2010, A\&A, 518, L3 Hansen, C. J., \& Kawaler, S. D. 1994, Stellar Interiors. Physical Principles, Structure, and Evolution, 84

Hanson, M. M., Kudritzki, R.-P., Kenworthy, M. A., Puls, J., \& Tokunaga, A. T. 2005, ApJS, 161, 154

Hildebrand, R. H. 1983, QJRAS, 24, 267

Hollenbach, D. J., \& Tielens, A. G. G. M. 1997, ARA\&A, 35, 179

Inoue, A. K. 2001, AJ, 122, 1788

Irwin, M. J., Lewis, J., Hodgkin, S., et al. 2004, in Optimizing Scientific Return for Astronomy through Information Technologies, eds. P. J. Quinn \& A. Bridger, Proc. SPIE, 5493, 411

Israel, F. P. 1978, A\&A, 70, 769

Jenkins, E. B. 2004, Origin and Evolution of the Elements, 336

Kauffmann, J., Pillai, T., Shetty, R., Myers, P. C., \& Goodman, A. A. 2010a, ApJ, 712, 1137

Kauffmann, J., Pillai, T., Shetty, R., Myers, P. C., \& Goodman, A. A. 2010b, ApJ, 716, 433

Koenig, X. P., \& Leisawitz, D. T. 2014, ApJ, 791, 131

Kurtz, S., Churchwell, E., \& Wood, D. O. S. 1994, ApJS, 91, 659

Lefloch, B., \& Lazareff, B. 1994, A\&A, 289, 559

Lequeux, J. 2005, The Interstellar Medium (Berlin: Springer)

Lockman, F. J. 1989, ApJS, 71, 469

Lucas, P. W., Hoare, M. G., Longmore, A., et al. 2008, MNRAS, 391, 136

Lumsden, S. L., Hoare, M. G., Oudmaijer, R. D., \& Richards, D. 2002, MNRAS, 336, 621

MacLaren, I., Richardson, K. M., \& Wolfendale, A. W. 1988, ApJ, 333, 821

Martins, F., \& Plez, B. 2006, A\&A, 457, 637

Marton, G., Tóth, L. V., Paladini, R., et al. 2016, MNRAS, 458, 3479

McKee, C. F., \& Ostriker, E. C. 2007, ARA\&A, 45, 565

Minniti, D., Lucas, P. W., Emerson, J. P., et al. 2010, New A, 15, 433

Molinari, S., Swinyard, B., Bally, J., et al. 2010, A\&A, 518, L100

Morales, E. F. E., Wyrowski, F., Schuller, F., \& Menten, K. M. 2013, A\&A, 560, A76

Oka, T., Onodera, Y., Nagai, M., et al. 2012, ApJS, 201, 14

Ossenkopf, V., \& Henning, T. 1994, A\&A, 291, 943

Panagia, N., \& Walmsley, C. M. 1978, A\&A, 70, 411

Price, S. D., Egan, M. P., Carey, S. J., Mizuno, D. R., \& Kuchar, T. A. 2001, AJ, 121,2819

Quireza, C., Rood, R. T., Bania, T. M., Balser, D. S., \& Maciel, W. J. 2006, ApJ, 653,1226

Saito, R. K., Hempel, M., Minniti, D., et al. 2012, A\&A, 537, A107

Samal, M. R., Zavagno, A., Deharveng, L., et al. 2014, A\&A, 566, A122

Schuller, F., Menten, K. M., Contreras, Y., et al. 2009, A\&A, 504, 415

Sharpless, S. 1959, ApJS, 4, 257

Siringo, G., Weiss, A., Kreysa, E., et al. 2007, The Messenger, 129, 2

Sternberg, A., Hoffmann, T. L., \& Pauldrach, A. W. A. 2003, ApJ, 599, 1333

Strömgren, B. 1939, ApJ, 89, 526

Strong, A. W., \& Mattox, J. R. 1996, A\&A, 308, L21

Sunada, K., Nakazato, T., Ikeda, N., et al. 2007, PASJ, 59, 1185

Urquhart, J. S., Hoare, M. G., Purcell, C. R., et al. 2009, A\&A, 501, 539

Urquhart, J. S., Morgan, L. K., Figura, C. C., et al. 2011, MNRAS, 418, 1689

Urquhart, J. S., Moore, T. J. T., Schuller, F., et al. 2013, MNRAS, 431, 1752

Vacca, W. D., Cushing, M. C., \& Rayner, J. T. 2003, PASP, 115, 389

Watson, C., Povich, M. S., Churchwell, E. B., et al. 2008, ApJ, 681, 1341

Whitworth, A. P., Bhattal, A. S., Chapman, S. J., Disney, M. J., \& Turner, J. A. 1994, MNRAS, 268, 291

Williams, J. P., de Geus, E. J., \& Blitz, L. 1994, ApJ, 428, 693

Wood, D. O. S., \& Churchwell, E. 1989, ApJ, 340, 265

Wright, E. L., Eisenhardt, P. R. M., Mainzer, A. K., et al. 2010, AJ, 140, 1868

Zavagno, A., Deharveng, L., Comerón, F., et al. 2006, A\&A, 446, 171

Zavagno, A., Russeil, D., Motte, F., et al. 2010, A\&A, 518, L81

Zinnecker, H., \& Yorke, H. W. 2007, ARA\&A, 45, 481 